\title{
INTERMEDIATE NORMALIZING EXTENSIONS ${ }^{1}$
}

\author{
BY
}

\author{
A. G. HEINICKE AND J. C. ROBSON
}

\begin{abstract}
Relationships between the prime ideals of a ring $R$ and of a normalizing extension $S$ have been studied by several authors recently. In this work, most of these known results are extended to give relationships between the prime ideals of $R$ and of $T$ where $T$ is a ring with $R \subset T \subset S$, and $S$ is a normalizing extension of $R$ : such rings $T$ are called intermediate normalizing extensions of $R$.

One result ("Cutting Down") asserts that for any prime ideal $J$ of $T, J \cap R$ is the intersection of a finite set of prime ideals $P_{i}$ of $R$, uniquely defined by $J$, whose corresponding factor rings $R / P_{i}$ are mutually isomorphic. The minimal members of the family of $P_{i}$ 's are the primes of $R$ minimal over $J \cap R$, and an "incomparability" theorem is proved which shows that no two comparable primes of $T$ can have their intersections with $R$ share a common minimal prime. Other results include versions of the "lying over" and "going up" theorems, proofs that chain conditions such as right Goldie or right Noetherian pass between $T / J$ and each of the rings $R / P_{i}$, and a demonstration that the "additivity principle" holds.
\end{abstract}

1. Introduction. Suppose that $R$ is a subring of $S$, sharing the same identity element, and that $S$ is finitely generated as an $R$-module by elements $a_{1}, \ldots, a_{n}$ with $a_{i} R=R a_{i}$. Then $S$ is called a normalizing extension of $R$. The relationship between the prime ideals of these two rings has been extensively studied, both by the authors $[5,6,7]$ and by Lorenz [11], Passman [13], Lanski [10] and others [2,3, 14, 17]. This relationship is very similar to that of Krull, and of Cohen and Seidenberg, for integral extensions of a commutative ring. Indeed, it is known [11, 1.3] that, in a sense, $S$ is integral over $R$.

This suggests that a similar relationship could exist between the prime ideals of $R$ and those of any ring $T$ with $R \subset T \subset S$, such a ring $T$ being termed an intermediate normalizing extension of $R$. In fact there are already results about this relationship when the $a_{i}$ centralize $R[15,16]$ and in some other special cases [9].

In this paper a description, almost as complete as that for normalizing extensions, is given of the relationship between the prime ideals of $R$ and any intermediate normalizing extension $T$. It is shown that, associated with any prime ideal $J$ of $T$, there is a set $\left\{P_{1}, \ldots, P_{k}\right\}$ of at most $n$ (the number of generators of $S$ ) prime ideals of $R$, uniquely defined by $J$; these are said to be connected to $J$. Moreover, as the "cutting down" theorem asserts, $\cap P_{i}=J \cap R$, and the prime rings $R / P_{i}$ are all isomorphic. It follows, of course, that $J \cap R$ is semiprime and has finitely many minimal primes.

Received by the editors March 15, 1983.

1980 Mathematics Subject Classification. Primary 16A56, 16A66; Secondary 16A26, 16A34, 16A55.

'Research was supported in part by a grant from the Natural Sciences and Engineering Research Council of Canada, Grant A-7338. 
An "incomparability" theorem is also proved. This says that no two comparable primes of $T$ can have the same intersection with $R$; in fact, their intersections cannot share a common minimal prime. This is obtained as a corollary to the "essentiality" theorem which proves that any nonzero ideal of $T / J$ is actually essential as an $R$-subbimodule of $T / J$. There are appropriate versions of "lying over" and "going up" theorems, the latter being new even when $S$ is a centralizing (liberal) extension of $R$. Also it is shown that chain conditions such as right Goldie or right Noetherian pass between $T / J$ and each of the rings $R / P_{i}$, and that the "additivity principle" holds.

In obtaining these results, the paper depends upon the ideas and results of the authors' earlier papers [5,7] concerning the relationship between $R$ and $S$, especially when $S$ is prime. In that case [7] found and used a particular collection of idempotent elements $\left\{f_{1}, \ldots, f_{m}\right\}$ in the Martindale quotient ring of $S$. These are used in similar fashion here to give, for each $i$, another ring $T_{i}$ which is linked with $T$ through a Morita context and with $T_{i} \supset f_{i} R \simeq R / P_{i}$. The general strategy is to reduce questions concerning $T$ to similar questions concerning $T_{i}$. There is a snag in dealing with the questions for $T_{i}$, since $T_{i}$ is not quite an intermediate normalizing extension of $f_{i} R$. Nevertheless, it is near enough for the techniques of [8] to be usable; and the results about $T_{i}$ make up an appendix to this paper.

The description of $T_{i}$ comes in $\S 2$ together with an analysis of the nature of $T / J$ as a $(T, R)$-bimodule. This culminates in the "cutting down" theorem. Similarly, $\S 3$ obtains "essentiality" and "incomparability" by conducting an analysis of $T / J$ as an $(R, R)$-bimodule. "Lying over" and "going up" come in $\S 4$, together with some similar results for prime ideals of $T$ and $S$. Easy examples show that, if $I$ is a prime ideal of $S, I \cap T$ need not be semiprime. But, as $\S 5$ shows, $T / I \cap T$ has at most $n$ minimal primes, and its prime radical is nilpotent of index at most $n$. This gives connections between the prime radicals of $R, T$ and $S$. Similar results for primitive ideals and the Jacobson radical also come in $\S \S 4$ and 5. Finally, chain conditions are discussed in $\S 6$.

It is surely clear from above that the papers $[5,7]$ are prerequisites for this paper. The relevant constructions and theorems will be described and quoted as appropriate.

2. Standard settings and cutting down. Suppose that $S$ is a normalizing extension of $R$, that $T$ is an intermediate normalizing extension with $R \subset T \subset S$, and that $J$ is a prime ideal of $T$. In studying $J$ and $T / J$, one can usually reduce problems to the case in which

(i) $S$ is a prime ring, and

(ii) $B \cap T \not \subset J$ whenever $0 \neq B \triangleleft S$;

this case being described as a standard setting for $J$. In this section the reduction is described, and then the structure of $T / J$ as a $(T, R)$-bimodule is deduced from the facts [7] concerning ${ }_{S} S_{R}$. This leads to the Cutting Down Theorem.

LEMMA 2.1. Let $R \subset T \subset S$ with $S$ a prime normalizing extension of $R$, and let $J$ be a prime ideal of $T$. This is a standard setting if and only if $J$ is not essential in ${ }_{R} T_{R}$ (i.e., $\left.J \notin E\left({ }_{R} T_{R}\right)\right)$. 
Proof. Corollary 2.25 of [7] shows that if ${ }_{R} W_{R}^{\prime} \subset{ }_{R} W_{R} \subset{ }_{R} S_{R}$ then $W^{\prime} \in \mathcal{E}\left({ }_{R} W_{R}\right)$ if and only if $W \cap B \subset W^{\prime}$ for some $0 \neq B \triangleleft S$. Take $W=T$ and $W^{\prime}=J$.

Proposition 2.2. Let $R \subset T \subset S$ with $S$ a normalizing extension of $R$, and let $J$ be a prime ideal of $T$. There is an ideal $I$ of $S$ with $I \cap T \subset J$ such that, with identifications of subrings,

$$
R / I \cap R \circlearrowright T / I \cap T \succ S / I
$$

gives a standard setting for $J / I \cap T$.

Proof. By Zorn's lemma, there is an ideal $I$ of $S$ maximal with respect to $I \cap T \subset J$. The inclusions above give $T / I \cap T$ as an intermediate extension of $R / I \cap R$, and give a standard setting for $J / I \cap T$, the verifications being routine.

NotAtion 2.3. For the study of $R / J \cap R$ and $T / J, 2.2$ makes clear that one may assume that the setting is standard for $J$ (and then $I$ is zero and $S$ is prime). Notation is fixed as such until specified otherwise (in the final few results).

Use will be made of results from [7] concerning the structure of $S$, and a brief survey of the relevant information follows.

First, $S$ embeds in $Q=Q(S)$, the Martindale right quotient ring of $S$. In $Q$ there is a family $f_{1}, \ldots, f_{m}$ of orthogonal idempotents which sum to 1 and which centralize $R$. The set $\left\{P_{i} \mid 1 \leqslant i \leqslant m\right\}$, with $P_{i}=\operatorname{ann}_{R}\left(f_{i}\right)$ is a set of $m$ (different) prime ideals of $R$ : these are the primes of $R$ connected to the zero ideal of $S$. Although proper inclusions may occur among the ideals $P_{i}, R / P_{i} \cong R / P_{j}$ for all $i$ and $j$.

Since $0=\cap_{1}^{m} P_{i}, R$ is semiprime and embeds into the subring $R^{*}$ of $Q$, where $R^{*}=\Sigma_{1}^{\oplus m} f_{i} R$, a direct product of the prime rings $f_{i} R\left(\cong R / P_{i}\right)$. Also $Q$ decomposes as $\sum_{i, j=1}^{\oplus m} f_{i} Q f_{j}$, this being a direct sum of $(R, R)$ and of $\left(R^{*}, R^{*}\right)$-bimodules. For any $W \triangleleft_{R} Q_{R}$, set $W_{i j}=W \cap f_{i} W f_{j}=W \cap f_{i} Q f_{j}$. Then $\sum_{i, j=1}^{m} W_{i j}$ is denoted by $W^{\circ}$, this being termed the interior of $W$. From [7, 4.5 and 4.6], $W^{\circ}$ is the largest $R^{*}$-subbimodule of $Q$ contained in $W$, and ${ }_{R} W_{R}^{\circ}$ is essential in ${ }_{R} W_{R}$.

Corollary 2.25 of [7] (cited in the proof of 2.1) can be applied to $T^{\circ} \subset T$, showing that there is a nonzero ideal $I_{0}$ of $S$ such that $I_{0} \cap T \subset T^{\circ}$. This notation will now be fixed. Since the setting is standard for $J$, then $I_{0} \cap T \not \subset J$.

For each idempotent $f_{i}$, the subsets $T_{i}=T_{i i}+f_{i} R=\left(T \cap f_{i} Q f_{i}\right)+f_{i} R$ and $S_{i}=$ $S_{i i}+f_{i} R$ are subrings of $Q$. There is a chain

$$
f_{i} R \subset T_{i} \subset S_{i} \subset f_{i} S f_{i} \subset f_{i} Q f_{i}
$$

and $f_{i} S f_{i}$ is a normalizing $f_{i} R$-bimodule, generated by the $f_{i} a_{k} f_{i}$ where $a_{1}, \ldots, a_{n}$ are $R$-normalizing generators of $S$. Furthermore, each $S_{i}$ is a prime ring with $Q\left(S_{i}\right) \simeq f_{i} Q f_{i}$ [7, 2.18]. This chain, with $T_{i}$ omitted, is studied in [7, Appendix A], and then a Morita context is used to relate $S$ with $S_{i}$.

Before doing likewise for $T$ and $T_{i}$, it is convenient to recall certain facts regarding Morita contexts whose proofs may be discovered in [1]. As noted in [7], given a Morita context $\left[\begin{array}{c}\Lambda \\ W\end{array}\right]$ r there is one-to-one correspondence between

$$
\mathscr{P}(\Lambda)=\{I \triangleleft \Lambda \mid I \text { prime and } V W \not \subset I\}
$$


and

$$
\mathscr{P}(\Gamma)=\left\{I^{\prime} \triangleleft \Gamma \mid I^{\prime} \text { prime and } W V \not \subset I^{\prime}\right\}
$$

given by $I \mapsto \theta(I)=\{\gamma \in \Gamma \mid V \gamma W \subset I\}$.

Proposition 2.4 [12, Lemma 4]. Let $I \in \mathcal{P}(\Lambda)$. Then $\Lambda / I$ is right nonsingular if and only if $\Gamma / \theta(I)$ is right nonsingular.

Next, suppose that $X_{\Lambda}$ is a prime module, which means that if $x I=0$ for $0 \neq x \in X$ and $I \triangleleft \Lambda$ then $X I=0$ (or, equivalently, that ann $X_{\Lambda}=$ ass $X_{\Lambda}$ ). It follows that ann $X$ is a prime ideal, $J$ say.

Lemma 2.5. Suppose that $X$ and $J$ are as above and $0 \neq x \in X$. Let $U=\{v \in V \mid$ $x v W=0\}$, a submodule of $V_{\Gamma}$.

(i) If $J \notin \mathscr{P}(\Lambda)$ then $V / U=0$;

(ii) if $J \in \mathscr{P}(\Lambda)$ then $(V / U)_{\Gamma}$ is a prime module with annihilator $\theta(J)$. Moreover, if $X_{\Lambda}$ is uniform, or simple, then so too is $(V / U)_{\Gamma}$.

Consider now the Morita context relating $T$ and $T_{i}$ given by

$$
\left[\begin{array}{cc}
T & T T^{\circ} f_{i} \\
f_{i} T^{\circ} T & T_{i}
\end{array}\right] .
$$

We write $\theta_{i}$ rather than $\theta$, and $\mathscr{P}_{i}(T)$ rather than $\mathscr{P}(T)$ when precision is required. The next result provides criteria, in a standard setting for $J$, for $J$ to belong to $\mathscr{P}_{i}(T)$.

Proposition 2.6. Let $T / K$ be a cyclic right $T$-module with $\operatorname{ann}(T / K)_{T}=J$. The following are equivalent.

(i) $T^{\circ} f_{i} T^{\circ} \subset J$ (i.e., $J \notin \mathscr{P}_{i}(T)$ ).

(ii) $T_{i i}{ }^{q} \subset J$ for some $q$.

(iii) $T_{i i} \subset J$.

(iv) $f_{i} T^{\circ} \subset J$.

(v) $T^{\circ} f_{i} \subset J$.

(vi) $\left(I_{0} \cap T\right) f_{i}\left(I_{0} \cap T\right) \subset J$.

(vii) $T^{\circ} f_{i} \subset K$.

Proof. Note that

$$
\left(I_{0} \cap T\right) f_{i} \subset T^{\circ} f_{i}=\sum_{j, k} T_{j k} f_{i}=\sum_{j} T_{j i} \subset T
$$

and similarly, $f_{i}\left(I_{0} \cap T\right) \subset f_{i} T^{\circ} \subset T$. Then the following implications are all clear: (vi) $\Leftarrow$ (i) $\Leftarrow\{($ iv) or (v) $\} \Rightarrow$ (iii) $\Rightarrow$ (ii). Further implications will be considered one by one. Set $D=\left(I_{0} \cap T\right) f_{i}\left(I_{0} \cap T\right) \triangleleft T$.

(ii) $\Rightarrow$ (vi). One gets $D^{q+1} \subset\left(I_{0} \cap T\right)\left(f_{i} T^{\circ} f_{i}\right)^{q}\left(I_{0} \cap T\right) \subset T T_{i i}{ }^{q} T \subset J$ and so $D \subset J$.

(vi) $\Rightarrow\{(\mathrm{iv})$ and (v) $\}$. Since $\left(I_{0} \cap T\right) f_{i} \subset T$ and $\left(I_{0} \cap T\right) \not \subset J$, then $\left(I_{0} \cap T\right) f_{i} \subset J$ and so $\left(I_{0} \cap T\right) f_{i} T^{\circ} \subset J$. Therefore $f_{i} T^{\circ} \subset J$. Similarly $T^{\circ} f_{i} \subset J$.

(v) $\Rightarrow$ (vii) is clear since $J \subset K$.

(vii) $\Rightarrow$ (vi). One has $D \subset T^{\circ} f_{i} T^{\circ} \subset K$, and so $D \subset$ ann $T / K=J$. 
COROllary 2.7. If $J \in \mathscr{P}_{i}(T)$, then $T_{i i} \not \subset \theta_{i}(J)$.

Proof. $T_{i i}^{3} \subset T T^{\circ} f_{i} T_{i i} f_{i} T^{\circ} T$ and $T_{i i}^{3} \not \subset J$. Therefore $T_{i i} \not \subset \theta_{i}(J)$.

LEMma 2.8. If $J \in \mathscr{P}_{i}(T)$ and $0 \neq I^{\prime} \triangleleft S_{i}$ then $I^{\prime} \cap T_{i} \not \subset \theta_{i}(J)$.

Proof. Suppose, to the contrary, that $I^{\prime} \cap T_{i} \subset \theta_{i}(J)$. Now if $I=\{s \in S \mid$ $\left.f_{i} S^{\circ} S s S S^{\circ} f_{i} \subset I^{\prime}\right\}$ then $I$ is an ideal of $S$ and $S_{i i} I^{\prime} S_{i i} \subset I$, as is easily verified. Since $T^{\circ} \subset S^{\circ}$ it follows that

$$
\left(f_{i} T^{\circ} T\right)(I \cap T)\left(T T^{\circ} f_{i}\right) \subset I^{\prime} \cap T \subset \theta_{i}(J)
$$

and so $I \cap T \subset J$. Since the setting is standard for $J, I=0$ and so $S_{i i} I^{\prime} S_{i i}=0$. But $S_{i}$ is prime and $S_{i i}$ is a nonzero ideal of $S_{i}[7,2.22]$. This provides a contradiction.

The next few results concern $T / J$, viewed as a $(T, R)$-bimodule.

Proposition 2.9. Let $X=T / K$ be a cyclic prime right $T$-module, with ann $X=J$. Set $X_{i}=\left(T T^{\circ} f_{i}+K\right) / K \subset T / K$. In the Morita context of 2.6

(i) if $J \notin \mathscr{P}_{i}(T)$ then $X_{i}=0$; and

(ii) if $J \in \mathscr{P}_{i}(T)$ then $X_{i}$ has a natural right $T_{i}$-module structure and, as such, is prime with annihilator $\theta_{i}(J)$. Furthermore, if $X_{T}$ is uniform, or simple, then so too is $\left(X_{i}\right)_{T_{i}}$.

Proof. Note that $X_{i} \simeq T T^{\circ} f_{i} /\left(T T^{\circ} f_{i} \cap K\right)$. Thus the result will follow directly from 2.5, applied to this Morita context, provided that, in the notation of 2.5, it is shown that $U=V \cap K$, where $V=T T^{\circ} f_{i}$ and $x=[1+K]$. In this case $U=\{v \in$ $\left.T T^{\circ} f_{i} \mid v f_{i} T^{\circ} \subset K\right\}$.

(i) Here $T^{\circ} f_{i} \subset J$, by 2.6 , and so $U=V=T T^{\circ} f_{i} \subset K$.

(ii) Again let $D=\left(I_{0} \cap T\right) f_{i}\left(I_{0} \cap T\right)$, and suppose $v \in U$.

Then $v D=v f_{i} D=v f_{i}\left(I_{0} \cap T\right) f_{i}\left(I_{0} \cap T\right) \subseteq v f_{i} T^{\circ} T \subset K$. But $D \not \subset J$, by 2.6, and $X$ is prime; so $v \in K$ and thus $U \subset V \cap K$. On the other hand, $\left(K \cap T T^{\circ} f_{i}\right) f_{i} T^{\circ} \subset$ $K T=K$ and so $V \cap K \subset U$.

The next result involves the prime ideals $P_{i}=\operatorname{ann}_{R} f_{i}$. As in 2.9,

$$
X_{i}=\left(T T^{\circ} f_{i}+K\right) / K \text {. }
$$

Proposition 2.10. Each $X_{i}$ is an $R$-submodule of $(T / K)_{R}$ with $X_{i} P_{i}=0$, and there is a chain of right $R$-module inclusions

$$
0 \neq\left(I_{0} \cap T\right)+K / K \subset \sum_{i=1}^{m} X_{i} \subset T / K .
$$

Proof. Since the setting is standard for $J$, and $0 \neq I_{0} \triangleleft S$, then $I_{0} \cap T \not \subset J=$ ann $T / K$. Therefore $I_{0} \cap T \not \subset K$ and so $0 \neq\left(I_{0} \cap T\right)+K / K$. It is easily checked that $X_{i}$ is a right $R$-submodule of $T / K$ and, moreover, that the right $R$-module structure inherited from $T / K$ coincides with that induced by the homomorphism $R \rightarrow f_{i} R \hookrightarrow T_{i}$ and the $T_{i}$-module structure of $X_{i}$. Therefore $X_{i} P_{i}=0$. It remains to show that the sum $\Sigma X_{i}$ is direct. Suppose $0=\Sigma_{1}^{m} x_{i}$ with $x_{i}=t_{i}+K$ and $t_{i} \in T T^{\circ} f_{i}$. Setting $y=\Sigma t_{i}$, then $y \in K$; so $K \supset y T_{i i}=t_{i} T_{i i}$ and hence $x_{i} T_{i i}=0$. If $J \in \mathscr{P}_{i}(T)$ 
then $x_{i} T_{i i}=0$ in the prime $T_{i}$ module $X_{i}$, yet, by $2.7, T_{i i} \not \subset \theta_{i}(J)=$ ann $X_{i}$, and thus $x_{i}=0$. On the other hand, if $J \notin \mathscr{P}_{i}(T)$ then $X_{i}=0$ and again $x_{i}=0$, as required.

Before completing the description of $X_{i}$, we recall from $[7,2.2]$ that $f_{i} S f_{i}$ is a prime right (and left) $R$-module with annihilator $P_{i}$ (i.e., in the terminology used there, $f_{i} S f_{i}$ is right (and left) torsion-free over $f_{i} R$ ).

Proposition 2.11. Suppose $J \in \mathscr{P}_{i}(T)$. Then

(i) $\theta_{i}(J) \cap f_{i} R=0$;

(ii) $X_{i}$ is a prime right $R$-module with annihilator $P_{i}$.

Proof. Note first that $T_{i} \subset f_{i} S f_{i}$ which is a torsion-free normalizing $f_{i} R$-bimodule. Therefore, if $0 \neq D \triangleleft f_{i} R$ then $[6,4.2] D T_{i}$ is an essential $f_{i} R$-subbimodule of $T_{i}$ and there exists $0 \neq D^{\prime} \triangleleft f_{i} R$ with $0 \neq T_{i} D^{\prime} \subset D T_{i}$.

(i) If $\theta_{i}(J) \cap f_{i} R \neq 0$ then taking this to be $D$, it follows from the above that $D T_{i} \cap T_{i i}$ is essential in $T_{i i}$ considered as an $R$-bimodule. By [7, 2.25], there exists $0 \neq I^{\prime} \triangleleft S$ with $I^{\prime} \cap T_{i i} \subset D T_{i} \cap T_{i i} \subset D T_{i}$, and then $0 \neq I_{i i}^{\prime} \triangleleft S_{i}[7,2.14]$ and $\left(I_{i i}^{\prime} \cap T_{i}\right) T_{i i} \subset I^{\prime} \cap T_{i i} \subset D T_{i} \subset \theta_{i}(J)$. This is impossible, by 2.7 and 2.8 , proving $\theta_{i}(J) \cap f_{i} R=0$.

(ii) Suppose that $0 \neq D \triangleleft f_{i} R$ and $x \in X_{i}$ with $x D=0$. With $D^{\prime}$ as above, $x T_{i} D^{\prime} T_{i} \subset x D T_{i}=0$. Since $X_{i}$ is a prime right $T_{i}$-module, $x=0$. This shows that $X_{i}$, considered as an $f_{i} R$-module, is torsion-free, which is readily seen as equivalent to (ii).

It is convenient to extract some information for the case where the setting is not standard.

THEOREM 2.12. Let $T$ be any intermediate normalizing extension of $R, T / K$ a cyclic prime right $T$-module, and $J=\operatorname{ann}(T / K)$.

(a) There exists a finite set $Y_{1}, \ldots, Y_{k}$ of right $R$-submodules of $T$ and an ideal $J_{0}$ of $T$ such that

(i) $Y_{i} \underset{\neq}{\supset} K$ for each $i$,

(ii) $0 \neq J_{0}+K / K \subset \Sigma_{1}^{\oplus k}\left(Y_{i} / K\right) \subset T / K$, and

(iii) each $Y_{i} / K$ is a prime right $R$-module.

(b) If $K=J$, then each $Y_{i}$ is a $(T, R)$-subbimodule of $T$ and $\Sigma^{\oplus} Y_{i} / J$ is an essential subbimodule of ${ }_{T}(T / J)_{R}$.

Proof. (a) If the setting were standard for $J$, the nonzero $X_{i} \subset T / K$ of $2.9-2.11$ have inverse images in $T$ which can be chosen for the $Y_{i}$, and $J_{0}=I_{0} \cap T$ is as required. If the setting is not standard, it can be made so, using 2.2; and then appropriate modules may be chosen in the factor rings, with inverse images in $T$ being as required.

(b) The first statement is clear from the construction of $Y_{i}$; and the second is trivial because $0 \neq J_{0}+J / J \triangleleft T / J$ and so $J_{0}+J / J$ is essential in $T_{T}(T / J)_{R}$.

This now gives the first of the connections between primes of $R$ and $T$.

THEOREM 2.13 (CUTTING DOWN). Let $T$ be an intermediate normalizing extension of $R$ and $J$ a prime ideal of $T$. Then $J \cap R$ is a finite intersection of prime ideals with mutually isomorphic factor rings. 
Proof. With the notation of 2.12(a), in the case when $K=J$, it is clear that rt. ann. $T\left(J_{0}+J / J\right)=$ rt. ann. $T(T / J)=J$ and so

$$
J \cap R=\text { rt.ann. }{ }_{R}\left(\sum_{1}^{k}\left(Y_{i} / J\right)\right)=\bigcap_{1}^{k} \mathrm{rt.ann}_{R}\left(Y_{i} / J\right) .
$$

But rt. ann. ${ }_{R}\left(Y_{i} / J\right)$ is a prime ideal, also by $2.12(\mathrm{a})$. The isomorphism of factor rings follows from the corresponding result $[5,2.12]$ for the prime ideal $I$ of $S$ ( $I$ being chosen as in 2.2), since each rt. ann. ${ }_{R}\left(Y_{i} / J\right)$ is one of the prime ideals obtained from $I$ (i.e., a prime ideal in conn ${ }_{R} I$ ).

REMARK. Since $\Sigma^{\oplus} Y_{i} / J$ is essential in ${ }_{T}(T / J)_{R}$, by $(2.12)$, it is easy to show that if ${ }_{T} V_{R}$ is any subbimodule of $T / J$ with $V_{R}$ a prime module, then rt.ann. ${ }_{R}(V)$ is one of the ideals rt.ann. ${ }_{R}\left(Y_{i} / J\right)$. This observation shows that the set of primes rt. ann. ${ }_{R}\left(Y_{i} / J\right)$ constructed in 2.12 is independent of which normalizing extension $S$ contains $T$, and of the choices of $I$, the idempotents $f_{i}$, etc. Indeed, this set is also independent of the choice to consider $(T / J)_{R}$ rather than ${ }_{R}(T / J)$ : after reducing to a standard setting for $J$ the set of primes obtained, on the left or right, is simply $\left\{P_{i} \mid T_{i i} \not \subset J\right\}=\left\{P_{i} \mid J \in \mathscr{P}_{i}(T)\right\}$. This proves

PROPOSITION 2.14. (i) The prime ideals of $R$ obtained from $J$ in 2.13 are independent of the choices made in their construction.

(ii) If $I$ is chosen as in 2.2, these primes are a subset of $\operatorname{conn}_{R}(I)$.

Definition 2.15. The primes described above are said to be connected to $J$ (and vice-versa); and the set of primes will be denoted by $\operatorname{conn}_{R}(J)$. The minimal members of this set then comprise $\operatorname{link}_{R}(J)$; they are the minimal primes of the semiprime ideal $J \cap R$ and are said to be linked to $J$. This description means that $\operatorname{link}_{R}(J)$ is independent of choices too.

3. Incomparability and essentiality. This section studies the $(R, R)$-bimodule structure of $T / J$, and uses this to obtain the Essentiality Theorem and its consequence, the Incomparability Theorem.

Again it will be supposed, until specified otherwise, that $R \subset T \subset S$ provides a standard setting for the prime ideal $J$ of $T$. Relabelling the idempotents $f_{i}$ if necessary, we may assume that $\operatorname{conn}_{R} J$ consists of $\left\{P_{1}, \ldots, P_{k}\right\}$ with $k \leqslant m$. Thus $J \in \mathscr{P}_{i}(T)$ if and only if $1 \leqslant i \leqslant k$. To simplify notation, we write $J_{i}$ rather than $\theta_{i}(J)$; so $J_{i}$ is a proper prime ideal of $T_{i}$ if $1 \leqslant i \leqslant k$, and $J_{i}=T_{i}$ if $k<i \leqslant m$. The first result is similar to 2.10 , and uses the decomposition $T^{\circ}=\sum_{i, j=1}^{\oplus m} T_{i j}$.

LEMMA 3.1. The sum $\sum_{i, j=1}^{k}\left(T_{i j}+J / J\right)$ is a direct sum of $(R, R)$-subbimodules of $T / J$, and

$$
0 \neq\left(I_{0} \cap T+J\right) / J \subset \sum_{i, j=1}^{k}\left(T_{i j}+J / J\right)=\left(T^{\circ}+J\right) / J \subset T / J .
$$

Proof. Clearly $I_{0} \cap T+J \subset T^{\circ}+J=\sum_{i, j=1}^{m} T_{i j}+J$. From 2.6 it follows that if $i>k$ then $T_{i j}+T_{j i} \subset J$ for all $j$; so $T^{\circ}+J=\sum_{i, j=1}^{k} T_{i j}+J$. The remainder of the proof is similar to that of 2.10 , and so is omitted. 
Consider now the prime rings $T / J$ and $\left(T^{\circ}+J\right) / J$. Since they both contain $\left(I_{0} \cap T+J\right) / J$ as an ideal, these rings have the same Martindale right quotient ring.

Proposition 3.2. Each $f_{i}$ is associated with an idempotent $\bar{f}_{i}$ of $Q(T / J)$ such that

(i) $\bar{f}_{i} \neq 0$ if and only if $1 \leqslant i \leqslant k$;

(ii) $\left\{\bar{f}_{i} \mid 1 \leqslant i \leqslant k\right\}$ is a family of orthogonal idempotents summing to 1 in $Q(T / J)$;

(iii) for $1 \leqslant i \leqslant k, \bar{f}_{i}$ centralizes $R$ and $\operatorname{ann}_{R}\left(\bar{f}_{i}\right)=P_{i}$.

Proof. For each $i$, the restriction to $\left(I_{0} \cap T+J\right) / J$ of the map $\left(T^{\circ}+J\right) / J \rightarrow$ $\left(T^{\circ}+J\right) / J$ defined by $t+J \mapsto f_{i} t+J$ gives rise to $\bar{f}_{i}$ in $Q(T / J)$. Clearly $\bar{f}_{i} \bar{f}_{j}=0$ (when $i \neq j$ ) or $\bar{f}_{i}$ (when $i=j$ ), and $\bar{f}_{1}+\bar{f}_{2}+\cdots+\bar{f}_{m}$ is the identity in $Q(T / J)$. Now $\bar{f}_{i}=0$ if and only if $f_{i}\left(I_{0} \cap T \cap A\right) \subset J$ for some $A \triangleleft T$ with $J \subsetneq A$. It now follows easily from 2.6 that $\bar{f}_{i}=0 \Leftrightarrow f_{i}\left(I_{0} \cap T\right) A \subset J \Leftrightarrow J \notin \mathscr{P}_{i}(T)$ (i.e., when $i>k$ ). Thus (i) and (ii) are established.

(iii) Since $Q(T / J)$ is a ring, it can be regarded as a bimodule over $T / J$, hence over $R / J \cap R$, and thus as an $(R, R)$-bimodule. Since each $f_{i}$ centralizes $R$, so does $\bar{f}_{i}$. When $\bar{f}_{i} \neq 0, T_{i i}+J / J$ is right (and left) torsion-free over $f_{i} R$, by 2.11 (and its left-right dual), and this easily implies ann ${ }_{R}\left(\bar{f}_{i}\right)=P_{i}$.

For the rest of this section, unless otherwise specified, $i$ is in $\{1, \ldots, k\}$, so $J \in \mathscr{P}_{i}(T)$. Next we relate $\bar{f}_{i} Q(T / J) \bar{f}_{i}$ to $T_{i} / J_{i}$. Recall that $T_{i}, J$ and $J_{i}$ are all subsets of $Q(S)$.

Proposition 3.3. (i) $T_{i i}+J / J \cong T_{i i}+J_{i} / J_{i}$ as rings and as $(R, R)$-bimodules; (ii) this isomorphism extends to an isomorphism $\bar{f}_{i} Q(T / J) \bar{f}_{i} \cong Q\left(T_{i} / J_{i}\right)$.

Proof. (i) Recalling that $J_{i}=\left\{x \in T_{i} \mid T^{\circ} f_{i} x f_{i} T^{\circ} \subset J\right\}$, it is evident that $T_{i i} \cap J \subset$ $T_{i i} \cap J_{i}$. Furthermore, since $T_{i i} \cap J_{i}$ is a subset of $T$ with $\left(I_{0} \cap T\right)\left(T_{i i} \cap J_{i}\right)\left(I_{0} \cap T\right)$ $\subset T^{\circ} f_{i} J_{i} f_{i} T^{\circ} \subset J$, it follows that $T_{i i} \cap J=T_{i i} \cap J_{i}$, and now (i) follows easily.

(ii) It is sufficient to show how $\bar{f}_{i} Q(T / J) \hat{f}_{i}$ can be identified as the Martindale quotient ring of $T_{i i}+J / J$. For $q \in Q(T / J)$, there exists $0 \neq A / J \triangleleft T / J$ with $\bar{f}_{i} q \bar{f}_{i}(A / J) \subset f_{i} T^{\circ}+J / J$, so multiplication on the left by $\bar{f}_{i} q \bar{f}_{i}$, when restricted to $\left(f_{i}\left(I_{0} \cap T\right) A\left(I_{0} \cap T\right) f_{i}+J\right) / J$ induces an element $\tilde{q}$ of $Q\left(T_{i i}+J / J\right)$. If $\tilde{q}=0$ then $\bar{f}_{i} q \bar{f}_{i} X=0$ for some ideal $X / J \neq 0$ in $T_{i i}+J / J$, and then

$$
\bar{f}_{i} q \bar{f}_{i}\left[\left(I_{0} \cap T\right) A\left(I_{0} \cap T\right) X\left(I_{0} \cap T\right)+J / J\right]=0,
$$

so $\bar{f}_{i} q \bar{f}_{i}=0$ in $Q(T / J)$. Thus $\bar{f}_{i} Q(T / J) \bar{f}_{i}$ embeds in $Q\left(T_{i i}+J / J\right)$, and this embedding is a ring homomorphism.

To see this is an isomorphism, let $q \in Q\left(T_{i i}+J / J\right)$, with $q(X / J) \subset T_{i i}+J / J$ where $J \subset X \triangleleft T_{i i}+J$. Set $Y=\left(I_{0} \cap T\right)\left(T_{i i}+J\right) X$ (so that $f_{i} Y \subset X$ ) and $Z=$ $Y\left(T_{i i}+J^{\prime}\right)\left(I_{0} \cap T\right)$. Then $Z \triangleleft T, Z \not \subset J$. We shall define a map $\hat{q}: Z / J \rightarrow T / J$.

Any $z$ in $Z$ may be written as a finite sum $\Sigma_{j} y_{j} t_{j} x_{j}$ with $y_{j} \in Y, t_{j} \in T_{i i}+J$ and $x_{j} \in I_{0} \cap T$ : then the image under $\hat{q}$ of $z+J$ is defined to be the coset $\hat{z}=$ $\sum_{j} q\left(f_{i} y_{j}+J\right) t_{j} x_{j}$. To see that this is well defined, note that if $z \in J$ then

$$
f_{i} z\left(I_{0} \cap T\right) \subset T \text { and }\left(I_{0} \cap T\right) f_{i} z\left(I_{0} \cap T\right) \subset J,
$$


whence $f_{i} z\left(I_{0} \cap T\right) \subset J$. Then, for every $v \in\left(I_{0} \cap T\right)\left(T_{i i}+J\right)$, each $t_{j} x_{j} v \in T_{i i}+J$ and so

$$
\hat{z} v=\sum_{j} q\left(f_{i} y_{j}+J\right) t_{j} x_{j} v=q\left(f_{i} z v+J\right)=q(J)=0 .
$$

Therefore, as an element of $T / J, \hat{z}$ annihilates $\left(I_{0} \cap T\right)\left(T_{i i}+J\right)$ and thus $\hat{z}=0$. Hence $\hat{q}$ is a well-defined function from $Z / J \rightarrow T / J$ : trivially it is a right $T$-module homomorphism, and it is easily verified that $\bar{f}_{i} \hat{q} \bar{f}_{i}$ corresponds to $q$ in the embedding of $\bar{f}_{i} Q(T / J) \bar{f}_{i}$ into $Q\left(T_{i i}+J / J\right)$.

REMARK 3.4. Under the isomorphism $\bar{f}_{i} Q(T / J) \bar{f}_{i} \cong Q\left(T_{i} / J_{i}\right)$, the subring $\bar{f}_{i} R+$ $\left(T_{i i}+J / J\right)$ is isomorphic to $T_{i} / J_{i}$, since $T_{i i}+J / J \cong T_{i i}+J_{i} / J_{i}$, and we have simply adjoined the $R$-submodule generated by the identity element in each ring.

Proposition 3.5. If $J \subset W \triangleleft_{R}\left(T^{\circ}+J\right)_{R}$ with $W / J$ essential in ${ }_{R}\left(T^{\circ}+J / J\right)_{R}$, then

(i) $W$ is essential in ${ }_{R} T_{R}$ and

(ii) there exists $0 \neq B \triangleleft S$ with $B \cap T \subset W$.

Proof. It is standard that $W$ must be essential in $T^{\circ}+J$; and $T^{\circ}$ essential in $T$, so (i) is proved. Now [7, 2.25] gives (ii).

Proposition 3.6. $Q(T / J) \bar{f}_{i}$ is right torsion-free, and $\bar{f}_{i} Q(T / J)$ is left torsion-free, as an $R / P_{i}$-module.

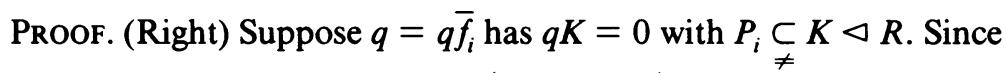

$$
\left(f_{i} T^{\circ} T+J\right) / J
$$

is left torsion-free over $f_{i} R$ (by the left-right dual of 2.10$),\left(K f_{i} T^{\circ}+J\right) / J$ is essential in $\left(f_{i} T^{\circ}+J\right) / J$, and so

$$
\left(K f_{i} T^{\circ}+J / J\right) \oplus\left(\sum_{\substack{j=1 \\ j \neq i}}^{k} f_{j} T^{\circ}+J / J\right)
$$

is essential in $T^{\circ}+J / J$. By 3.5,

$$
B \cap T \subset K f_{i} T^{\circ}+\left(\sum_{\substack{j=1 \\ j \neq i}}^{k} f_{j} T^{\circ}\right)+J \quad \text { for some } 0 \neq B \triangleleft S .
$$

Then

$$
\begin{aligned}
q(B \cap T+J / J)= & q \bar{f}_{i}(B \cap T+J / J) \\
& \subset q \bar{f}_{i}\left(K f_{i} T^{\circ}+J / J\right)+q \bar{f}_{i}\left(\sum_{\substack{j=1 \\
j \neq i}}^{k} f_{j} T^{\circ}+J / J\right)=0,
\end{aligned}
$$

and so $q$ is zero in $Q(T / J)$.

(Left) Suppose $q=\bar{f}_{i} q$ satisfies $K q=0$ with $P_{i} \subset K \triangleleft R$. Since $q=\bar{f}_{i} q, q D \subset f_{i} T^{\circ}$ $+J / J$ for an appropriately chosen ideal $D \neq 0$ of $T^{\circ}+J / J$. Then $K q D=0$, with 
$q D \subset f_{i} T^{\circ}+J / J$, implies $q D=0$ since $f_{i} T^{\circ}+J / J$ is left torsion-free over $f_{i} R$. Hence $q=0$.

The next observation concerns the relationship between $T_{i}$ and $f_{i} R$. The proof, which requires an analysis of subrings of $Q\left(S_{i}\right)$, is deferred to Appendix B (see B.5 of the Appendix).

Lemma 3.7. $J_{i}$ is maximal among ideals $J^{\prime}$ of $T_{i}$ with $J^{\prime} \cap f_{i} R=0$.

COROllaRY 3.8. Any nonzero ideal of $\left(T_{i i}+J / J\right)+\bar{f}_{i} R$ meets $\bar{f}_{i} R$ nontrivially.

Proof. The proof is immediate from 3.7 and 3.4 .

Proposition 3.9. With $Q^{\prime}$ denoting $Q(T / J)$, suppose $0 \neq q=\bar{f}_{i} q \bar{f}_{j} \in \bar{f}_{i} Q^{\prime} \bar{f}_{j}$ and suppose $0 \neq A \triangleleft T / J$. Then $R q R \cap A \neq 0$.

Proof. Choose $0 \neq B \triangleleft T / J$ with $q B \subset T / J$. Then, with $T_{i i}^{\prime} \operatorname{denoting}\left(T_{i i}+J\right) / J$,

$$
T_{i i}^{\prime} q\left(B A \cap \bar{f}_{j} Q^{\prime} \bar{f}_{j}\right) \subset A \cap Q^{\prime} \bar{f}_{j} \cap \bar{f}_{i} Q^{\prime}=A \cap \bar{f}_{i} Q^{\prime} \bar{f}_{j} .
$$

Now, by 3.8, $T_{i i}^{\prime} \cap \bar{f}_{i} R=\bar{f}_{i} H \neq 0$ for some $H \triangleleft R$ and $T_{j j}^{\prime} B A T_{j j}^{\prime} \cap \bar{f}_{j} R=\bar{f}_{j} K \neq 0$ for some $K \triangleleft R$. Noting that $\bar{f}_{j} K \subset B A \cap \bar{f}_{j} Q^{\prime} \bar{f}_{j}$, and using 3.6, it follows that

$$
0 \neq H q K=H \bar{f}_{i} q \bar{f}_{j} K \subset T_{i i}^{\prime} q\left(B A \cap \bar{f}_{j} Q^{\prime} \bar{f}_{j}\right) \subset A \cap \bar{f}_{i} Q^{\prime} \bar{f}_{j},
$$

as desired.

Next comes the main result of this section.

THEOREM 3.10 (ESSENTIALITY). Let $T$ be an arbitrary intermediate normalizing extension of a ring $R$. Let $J$ be a prime ideal of $T$ and $0 \neq A \triangleleft T / J$. Then $A$ is essential in ${ }_{R} Q(T / J)_{R}$ and in ${ }_{R}(T / J)_{R}$.

Proof. Let $Q^{\prime}=Q(T / J)$; so $Q^{\prime}=\sum_{i, j=1}^{\oplus k} \bar{f}_{i} Q^{\prime} \bar{f}_{j}$ by 3.2. By 3.9, $A \cap \bar{f}_{i} Q^{\prime} \bar{f}_{j}$ is essential in $\bar{f}_{i} Q^{\prime} \bar{f}_{j}$ for all $i, j$, from which it is standard that $A$ is essential in ${ }_{R} Q_{R}^{\prime}$ and hence in ${ }_{R}(T / J)_{R}$.

COROLlaRY 3.11. Let $S$ provide a standard setting for $J$. Then, for $W \triangleleft_{R} T_{R}$ with $W \supset J$, the following are equivalent:

(i) $W / J$ is essential in ${ }_{R}(T / J)_{R}$.

(ii) $W \supset J_{1}$ for some $J_{1} \triangleleft T, J_{1} \not \subset J$.

(iii) $W \supset B \cap T$ for some $0 \neq B \triangleleft S$.

Proof. (iii) $\Rightarrow$ (ii) is clear; (ii) $\Rightarrow$ (i) follows from 3.10 , and (i) $\Rightarrow$ (iii) follows from 3.5 .

COROLLARY 3.12 (INCOMPARABILITY). Let $T$ be an arbitrary intermediate normalizing extension of $R$, and let $J, J^{\prime}$ be ideals of $T$ with $J$ prime and $J \subset J^{\prime}$. Then $J \cap R \subset J^{\prime} \cap R$; indeed $J^{\prime} \cap R$ is not contained in any prime ideal of $R$ minimal over $J \cap R$.

Proof. $J_{1} / J$ is essential in ${ }_{R}(T / J)_{R}$ and so $\left(J_{1} \cap(R+J)\right) / J$ is essential in $R+J / J$. Let $P$ be a minimal prime over $J \cap R$; so $P / J \cap R$ is not essential in $R / J \cap R$. Therefore $J+P / J$ is not essential in $R+J / J$. Hence $J+\left(J_{1} \cap R\right)=$ $(R+J) \cap J_{1} \not \subset J+P$, and so $J_{1} \cap R \not \subset P$. 
We conclude this section with an observation about $\operatorname{conn}_{R}(J)$. This set was seen in $\S 2$ to consist of all ideals of the form rt. ann. ${ }_{R}(V)$ where ${ }_{T} V_{R}$ is a subbimodule of $T / J$ which is prime when considered as a right $R$-module. In fact, $\operatorname{conn}_{R}(J)$ can be identified solely in terms of $(T / J)_{R}$. To see this, define as $M$, the set of associated primes of an arbitrary module $M_{\Lambda}$, to be the set of all ann ${ }_{\Lambda} N$ for which $N_{\Lambda}$ is a (nonzero) prime submodule of $M$.

Proposition 3.13. $\operatorname{conn}_{R}(J)=\operatorname{ass}(T / J)_{R}=\operatorname{ass}_{R}(T / J)$.

Proof. It may be assumed that $S$ provides a standard setting for $J$. If $P_{i} \in$ $\operatorname{conn}_{R}(J)$ then $T_{i i}+J / J$ is a prime right $R$-module with annihilator $P_{i}$, so $P_{i} \in$ $\operatorname{ass}(T / J)_{R}$. Conversely, let $P \in \operatorname{ass}(T / J)_{R}$, and suppose $P=\operatorname{ann}_{R}(V)$ with $V_{R}$ a prime submodule of $T / J$. As a submodule of $Q(T / J)_{R}, V \subset \Sigma_{1}^{\oplus k} \bar{f}_{i}$. If $j \in\{1, \ldots, k\}$ is minimal with respect to $V \cap\left(\sum_{1}^{j} V \bar{f}_{i}\right) \neq 0$, then this intersection is a nonzero submodule of $V_{R}$ which embeds into $Q(T / J) \bar{f}_{j} R$. Since $V$ and $Q(T / J) \bar{f}_{j}$ are prime right $R$-modules, it follows that $P=P_{j}$ and thus conn ${ }_{R}(J)=\operatorname{ass}(T / J)_{R}$. The other assertion has a similar proof.

4. Lying over and going up. In this section analogues of the Cohen-Seidenberg "lying over" and "going up" theorems are established for prime ideals of $R$ and $T$. Then results related to maximum ideals and primitive ideals are obtained.

THEOREM 4.1 (LYING OVER). Let $T$ be an intermediate normalizing extension of $R$, and let $P$ be a prime ideal of $R$. Then

(i) $T$ contains an ideal $J$ maximal with respect to $J \cap R \subset P$;

(ii) any such ideal $J$ is prime, and $P$ is a minimal prime over $J \cap R$.

Proof. (i) is a routine application of Zorn's lemma, and $J$ is easily seen to be prime. To prove the rest of (ii) we may assume without loss of generality that $J$ is in a standard setting. If $P$ is not a minimal prime of $J \cap R$ then $P / J \cap R$ is essential in ${ }_{R}(R / J \cap R)_{R}$, and if $C$ is a complement of $R+J / J$ in ${ }_{R}(T / J)_{R}$, then $(P+J / J) \oplus$ $C$ is essential in ${ }_{R}(T / J)_{R}$. By 3.11, $S$ contains an ideal $B$ with $B \cap T \not \subset J$ but $(B \cap T+J) / J \subset(P+J / J) \oplus C$. Then $(R+J) \cap(B \cap T+J) \subset P+J$ and so $R \cap(B \cap T+J)=R \cap(R+J) \cap(B \cap T+J) \subset R \cap(P+J)=P$, contradicting the maximality of $J$.

Corollary 4.2 (GoING UP). Let $P_{1} \subset P_{2}$ be prime ideals of $R$ and $J_{1}$ a prime ideal of $T$ with $J_{1} \cap R \subset P_{1}$. Then there exists a prime ideal $J_{2}$ of $T$ with $J_{1} \subset J_{2}$ and $P_{2} a$ minimal prime over $J_{2} \cap R$.

Even in the case where $S$ is a finite centralizing (or liberal) extension of $R$, this result is new, answering a question left open in [15].

It is useful to have some similar results for the primes of $T$ and of $S$. If $J$ is a prime ideal of $T$ and $I$ is an ideal of $S$ maximal with respect to $I \cap T \subset J$ (as in 2.2) let us say that $J, I$ form a standard pair.

THEOREM 4.3 (LYING OVER; $T$ AND $S$ ). Let $R \subset T \subset S$ with $S$ a normalizing extension of $R$, and let $J$ be a prime ideal of $T$. Then there is a standard pair $J, I$ with $I$ a prime ideal of $S$; and $J$ is a minimal prime over $I \cap T$. 
Proof. Only the last statement needs proof; and for it we may assume $I=0$, using 2.2. Suppose that $J^{\prime}$ is a prime ideal of $T$ with $J^{\prime} \subset J$. Pick any idempotent $f_{i}$ (as in §2) with $J \in \mathscr{P}_{i}(T)$. Then evidently $J^{\prime} \in \mathscr{P}_{i}(T)$. Since $\theta_{i}\left(J^{\prime}\right) \subset \theta_{i}(J)$ and $\theta_{i}(J) \cap f_{i} R=0$, then 3.7 applied to $\theta_{i}\left(J^{\prime}\right)$ gives $\theta_{i}\left(J^{\prime}\right)=\theta_{i}(J)$ and so $J^{\prime}=J$. Thus $J$ is minimal.

COROllary 4.4 (GoIng UP; $T$ AND $S$ ). Let $J_{1} \subset J_{2}$ be prime ideals of $T$, and $I_{1} a$ prime ideal of $S$ with $I_{1} \cap T \subset J_{1}$. Then there exists a prime ideal $I_{2}$ of $S$ with $I_{1} \subset I_{2}$ and $J_{2}$ minimal over $I_{2} \cap T$.

Next, we investigate the relationship between prime ideals $P$ and $J$ where $P$ is a minimal prime over $J \cap R$, calling such a pair a linked pair.

THEOREM 4.5. If $T$ is an intermediate normalizingextension of $R$ and $P, J$ is a linked pair, then $P$ is a maximal ideal of $R$ if and only if $J$ is a maximal ideal of $T$.

Proof. If $J$ is maximal then 4.2 shows that $P$ is maximal. Conversely suppose $P$ is maximal. Using 2.2, we may suppose that the setting is standard for $J$. By 2.14(ii), $P$ must be connected to the zero ideal of $S$ and so, by [5, 5.11], $S$ must be a simple ring. Now 3.11 applies to show that $J$ is a maximal ideal of $T$.

Before obtaining a corresponding result for primitive ideals, an extension of a result of Formanek and Jategaonkar [4] (for normalizing extensions) is required.

THEOREM 4.6. Let $T$ be an intermediate normalizing extension of $R$ and $X$ a simple right $T$-module. Then $X_{R}$ is semisimple of finite length.

Without loss of generality, we may suppose that $R \subset T \subset S$ is a standard setting for $J=\operatorname{ann}_{T} X$, and that $X=T / K$, with $K$ a maximal right ideal. Application of 2.9-2.11 shows that $T / K=\sum_{i=1}^{\oplus m} X_{i}$ with each nonzero $X_{i}$ a simple right $T_{i}$-module with annihilator $\theta_{i}(J)=J_{i}$. This, together with 2.8 , shows that the chain $f_{i} R \subset T_{i} \subset$ $S_{i} \subset f_{i} S f_{i} \subset Q\left(S_{i}\right)$ is appropriate for the theory in Appendix B. Then B.12 shows that $X_{i}$ is semisimple of finite length as a right $f_{i} R$-module. Hence $(T / K)_{R}$ is semisimple of finite length.

THEOREM 4.7. If $T$ is an intermediate normalizing extension of $R$ and $P, J$ is a linked pair, then $P$ is right primitive if and only if $J$ is right primitive.

Proof. Suppose $J$ is right primitive, with $J=$ ann $X_{T}$ and $X_{T}$ simple. By 4.4, $X_{R}=\Sigma^{\oplus} W_{i}$ with each $\left(W_{i}\right)_{R}$ simple. Therefore $J \cap R=\cap \operatorname{ann}_{R} W_{i}$. Therefore $P=\operatorname{ann}_{R} W_{i}$ for some $i$, and so $P$ is right primitive.

Conversely, suppose $P$ is right primitive. Choose $I$ as in 2.2. By [5, 5.11], $I$ is right primitive, say $I=\operatorname{ann}_{S} Y$ with $Y$ a simple right $S$-module. By 4.6 (applied to $R$ and $S) Y_{R}$ is semisimple of finite length. Therefore $Y_{T}$ has finite length. If $Q_{1}, \ldots, Q_{k}$ are the annihilators of the composition factors, then $\Pi Q_{i} \subset \operatorname{ann}_{T} Y=I \cap T \subset J$. Thus, for some $i, Q_{i} \subset J$. Since the setting is standard for $J, 4.3$ shows that $J=Q_{i}$.

Let $\mathbf{J}$ denote the Jacobson radical, and $\mathbf{P}$ the prime radical.

COROllary 4.8. If $T$ is an intermediate normalizing extension of $R$ then $\mathbf{J}(R)=$ $\mathbf{J}(T) \cap R$ and $\mathbf{P}(R)=\mathbf{P}(T) \cap R$. 
Proof. These follow from "cutting down" (2.13) and "lying over" (4.1). The former also relies on 4.7 .

REMARK 4.9. Suppose that $J, I$ form a standard pair of primes in $T$ and $S$. It follows, from the results above, that $J$ is maximal if and only if $I$ is maximal, and $J$ is right primitive if and only if $I$ is right primitive. To see this, one simply chooses a prime ideal $P$ linked with $J$. Note that $P$ is connected to $I$ and so $R / P \simeq R / P^{\prime}$ for the minimal primes $P^{\prime}$ over $I \cap R$. Thus a double application of 4.5 or 4.7 (to $P, J$ and $\left.P^{\prime}, I\right)$ gives the claimed results.

A similar argument applies to the results of $\S 6$ (to come), but will not be explicitly pointed out each time.

5. Nilpotency. Easy examples, involving triangular matrix subrings of the examples described in [5 and 7] show that when $R \subset T \subset S$ as usual, and $I$ is a prime ideal of $S$, then $I \cap T$ need not be semiprime (as is $I \cap R$ ). This section provides some information about the nature of the ring $T / I \cap T$. As the examples would suggest, it has a prime radical which is nilpotent, with index at most the number $n$ of normalizing generators of $S$, and it has at most $n$ minimal primes. As indicated at the end of this section, there are some unanswered questions remaining.

First comes a result about prime normalizing extensions.

Proposition 5.1. Let $S$ be a prime normalizing extension of $R$, with $n$ normalizing generators. Let $X$ be both a nilpotent subring and an $R$-subbimodule of $S$. Then $X^{n}=0$.

Proof. Suppose that $X^{t}=0$. In $Q(S)$, the idempotents $f_{i}$ sum to 1 ; so $X^{j} \subset$ $\sum_{i=1}^{\oplus m} X^{j} f_{i}$ for each $j$. Thus it suffices to show $X^{n} f_{i}=0$ for each $i$.

Fixing $i$, and setting $X^{0}=S$, consider the chain $S f_{i}=X^{0} f_{i} \supset X f_{i} \supset X^{2} f_{i} \supset \cdots \supset$ $X^{t} f_{i}=0$ of subbimodules of the normalizing $\left(R, f_{i} R\right)$-bimodule $S f_{i}$. Let $k$ be the least integer for which $X^{k} f_{i} / X^{k+1} f_{i}$ is not faithful over $f_{i} R$. Then if $\rho$ is the rank $\rho_{f_{i} R}$ defined in [6, 3.1], $k \leqslant \rho\left(S f_{i}\right) \leqslant n$ by [6, 3.3 and 3.5]. Also, if $j \geqslant k$, then $X^{j} f_{i} D_{j} \subset$ $X^{j+1} f_{i}$ for some $D_{j} \triangleleft R$ with $P_{i} \subset D_{j}$, since $X^{j} f_{i} / X^{j+1} f_{i}$ is unfaithful. Therefore

$$
X^{k} f_{i} D_{k} D_{k+1} \cdots D_{t-1} \subset X^{t} f_{i}=0 \text {. }
$$

However, $0 \neq f_{i} D_{k} D_{k+1} \cdots D_{t-1}$ is an ideal of $f_{i} R$, and $S f_{i}$ is right torsion-free over $f_{i} R$. Therefore $X^{k} f_{i}=0$; and so $X^{n}=0$ as claimed.

THEOREM 5.2. Suppose that $T$ is an intermediate extension with $R \subset T \subset S$ and that $I$ is a prime ideal of $S$. Then the prime radical of $T / I \cap T$ is nilpotent of index at most $n$.

Proof. Without loss of generality, suppose $I=0$. Then any nilpotent ideal $X$ of $T$ satisfies $X^{n}=0$, by 5.1. A standard argument shows that the sum of all nilpotent ideals of $T$ is nilpotent, and coincides with the prime radical of $T$.

COROLlaRY 5.3. If $R \subset T \subset S$ is an arbitrary intermediate normalizing extension then $\mathbf{P}(T)^{n} \subset \mathbf{P}(S) \cap T \subseteq \mathbf{P}(T)$.

Proof. This follows directly from "lying over" (4.3) and 5.2.

There are corresponding results concerning the Jacobson radical. 
Proposition 5.4. Suppose that $R \subset T \subset S$ with $S$ a right primitive normalizing extension of $R$. Then $T$ has at most $n$ minimal primes, each of which is right primitive; and $\mathbf{J}(T)^{n}=0$.

Proof. The proof of 4.7 shows that the minimal primes of $T$ are amongst the primitive ideals $Q_{1}, \ldots, Q_{k}$, with $k \leqslant n$. Then $\mathbf{J}(T)=\mathbf{P}(T)$ and so $\mathbf{J}(T)^{n}=0$ by 5.2.

COROllaky 5.5. Let $R \subset T \subset S$ be an arbitrary intermediate normalizing extension. Then $\mathbf{J}(T)^{n} \subset \mathbf{J}(S) \cap T \subseteq \mathbf{J}(T)$.

Comparison of 5.2 and 5.4 suggests that, in the notation of 5.2, $I \cap T$ should have at most $n$ minimal primes. To show this, we use the "primitivity machine" of [13].

It is shown in [13] that given a ring, $K$ say, there is an overring $K^{\dagger}$ with the property that, if $P$ is any prime ideal of $K$, then $P^{\dagger}=P K^{\dagger}$ is a primitive ideal of $K^{\dagger}$. Other details required will be quoted as needed; but note that, simultaneously, one can form $R^{\dagger}, T^{\dagger}$ and $S^{\dagger}$; and the chain $R^{\dagger} \subset T^{\dagger} \subset S^{\dagger}$ gives again an intermediate normalizing extension, with $S^{\dagger}$ having $n$ normalizing generators over $R^{\dagger}$.

THEOREM 5.6. Suppose that $T$ is an intermediate normalizing extension with $R \subset T$ $\subset S$ and that $I$ is a prime ideal of $S$. Then there are at most $n$ primes of $T$ minimal over $I \cap T$.

Proof. Without loss of generality suppose $I=0$. Using the "primitivity machine", note that $S^{\dagger}$ is primitive and so, by 5.4, $T^{\dagger}$ has no more than $n$ minimal primes.

Let $\left\{Q_{\gamma} \mid \gamma \in \Gamma\right\}$ be the primes of $T$; so $\cap Q_{\gamma}=\mathbf{P}(T)$. By $[13,1.4], \cap Q_{\gamma}^{\dagger}=\mathbf{P}(T)^{\dagger}$ and, of course, each $Q_{\gamma}^{\dagger}$ is primitive. Thus $\mathbf{P}(T)^{\dagger}=\cap Q_{\gamma}^{\dagger} \supset \mathbf{P}\left(T^{\dagger}\right)$. On the other hand, $(\mathbf{P}(T))^{n}=0$ and so, by [13, 1.4], $\left(\mathbf{P}(T)^{\dagger}\right)^{n}=0$. Therefore $\mathbf{P}(T)^{\dagger} \subset \mathbf{P}\left(T^{\dagger}\right)$ and so $\mathbf{P}(T)^{\dagger}=\mathbf{P}\left(T^{\dagger}\right)$.

Now $T^{\dagger} / \mathbf{P}\left(T^{\dagger}\right)$ has at most $n$ minimal primes, say $H_{1}, \ldots, H_{h}$; and each is the annihilator of its complement. Moreover, $T^{\dagger} / \mathbf{P}\left(T^{\dagger}\right)=T^{\dagger} / \mathbf{P}(T)^{\dagger} \simeq(T / \mathbf{P}(T))^{\dagger}$. Therefore, by [13, 1.5], $H_{i} \cap(T / \mathbf{P}(T))=H_{i}^{\prime}$ say, is a prime ideal. And since $\cap H_{i}=0$, then $\cap H_{i}^{\prime}=0$. Thus $T / \mathbf{P}(T)$ has at most $n$ minimal primes.

This is a convenient point at which to comment on the parallels between the behaviours of the pairs $R, T$ and $T, S$. The results of this section show that, even though $I \cap T$ need not be semiprime (as is the case for $J \cap R$ ), nevertheless there are restrictions upon its prime radical. And, in both cases, there are at most $n$ minimal primes involved. Likewise the results of $\S 4$ give parallel results.

What remains unclear at present is the validity of an "incomparability" theorem for the pair $T, S$. This could be the statement:

(a) If $I$ and $I^{\prime}$ are ideals of $S$ with $I \subsetneq I^{\prime}$ with $I$ prime, and if $J$ is a minimal prime over $I \cap T$, then $I^{\prime} \cap T \not \subset J$.

It is not difficult, using the earlier results, to see that this is equivalent to each of the following statements:

(b) If $S$ is prime and $J$ is a minimal prime of $T$ then the setting is standard for $J$ (cf. 4.3). 
(c) If $S$ is prime and $J$ is a minimal prime of $T$ then $J$ is not essential in ${ }_{R} T_{R}$ (cf. 2.1).

The use of $S^{\dagger}$ again shows that it would suffice to prove any of these in the case when $S$ is primitive. Also it is perhaps worth noting that when $S$ is prime, the setting is standard for at least one of the minimal primes of $T$. (Otherwise one could deduce that $\mathbf{P}(T)$ is both nilpotent and essential in ${ }_{R} T_{R}$. This would contradict $R$ being semiprime.)

6. Chain conditions. Suppose that $R \subset T \subset S$ is an intermediate normalizing extension and that $P, J$ is a linked pair of prime ideals of $R$ and $T$. In this section we show that certain chain conditions pass between the two rings $R / P$ and $T / J$. Indeed, as noted in 4.9, they also pass between these two rings and the ring $S / I$, when $I$ is chosen as in 2.2. This notation is fixed henceforth.

Note first that both $R / I \cap R$ and $R / J \cap R$ are finite subdirect sums of rings isomorphic to $R / P$. So if one of $R / I \cap R, R / J \cap R$, and $R / P$ is right nonsingular, or right Goldie, or right Noetherian, then so are the other two.

THEOREM 6.1. Let $T$ be an arbitrary intermediate normalizing extension of $R$, and $P, J$ a linked pair of primes. Then $R / P$ is right nonsingular if and only if $T / J$ is right nonsingular.

Proof. Without loss of generality, we may suppose $I=0$. Using the notation of $\S 2$ and 3 , it follows that $P=P_{i}$ for one of the idempotents $f_{i}$ such that $J \in \mathscr{P}_{i}(T)$. Let $Z_{i}$ denote the inverse image in $T_{i}$ of the singular ideal of $T_{i} / J_{i}$, where $J_{i}=\theta_{i}(J)$. By 3.7 and B.15 (Appendix B), $f_{i} R$ is nonsingular if and only if $Z_{i}=J_{i}$. Thus $R / P$ is nonsingular if and only if $T_{i} / J_{i}$ is nonsingular, and so the result follows from 2.4 applied to the Morita context above 2.6.

Next it is shown that having a finite uniform dimension passes between $T / J$ and $R / P$. The second part of the following proof is similar to that of the comparable result for intermediate subrings of centralizing extensions, due to Stewart [16].

THEOREM 6.2. Let $T$ be an arbitrary intermediate normalizing extension of $R$, and $P, J$ a linked pair of primes. Then u. $\operatorname{dim} .(T / J)_{T}<\infty$ if and only if u. $\operatorname{dim} .(R / P)_{R}<$ $\infty$.

Proof. We may assume $R \subset T \subset S$ provides a standard setting for $J$, and that the idempotents $f_{i}$ are labelled so that $\operatorname{conn}_{R}(J)=\left\{P_{1}, \ldots, P_{k}\right\}$. Let $I_{0}$ denote the ideal of $S$ described in $\$ 2$.

(i) Assume that u. $\operatorname{dim} .(T / J)_{T}<\infty$. For each $i$ with $1 \leqslant i \leqslant k$, there is, by 3.2 and 3.4, a Morita context

$$
\left[\begin{array}{cc}
T / J & \left(T T^{\circ}+J / J\right) \bar{f}_{i} \\
\bar{f}_{i}\left(T^{\circ} T+J / J\right) & T_{i} / J_{i}
\end{array}\right] .
$$

By [1, Theorem 2], u. $\operatorname{dim} .\left(T_{i} / J_{i}\right)_{T_{i}}=$ u. $\operatorname{dim} . \bar{f}_{i}\left(T^{\circ} T+J / J\right)_{T} \leqslant$ u. $\operatorname{dim} .(T / J)_{T}$. Now B.13 of Appendix B gives u. $\operatorname{dim} .\left(f_{i} R_{f_{t} R}\right)<\infty$. Since $R / P \cong f_{i} R,(R / P)_{R}$ has finite uniform dimension. 
(ii) Now assume u. $\operatorname{dim} .(R / P)_{R}$ is finite. It will be shown that u. $\operatorname{dim} .(T / J)_{T}=$ u. $\operatorname{dim} .\left(I_{0} \cap T+J / J\right)_{T} \leqslant$ u. $\operatorname{dim} .\left(I_{0} \cap T+J / J\right)_{R} \leqslant n^{3}\left(\right.$ u. $\left.\operatorname{dim} .(R / P)_{R}\right)$, and clearly only the last inequality needs proof. From 3.1,

$$
I_{0} \cap T+J / J \subset \sum_{i, j=1}^{k} T_{i j}+J / J \subset T / J .
$$

Setting $X_{i j}=T_{i j}+J / J$, the sum $\sum_{i, j=1}^{\oplus k} X_{i j}$ is a direct sum of $(R, R)$-bimodules. It is clearly sufficient to prove that u. $\operatorname{dim} .\left(X_{i j}\right)_{R} \leqslant n\left[\mathrm{u} . \operatorname{dim} .(R / P)_{R}\right]$ whenever $i, j \leqslant k$.

Note that $f_{i} R \cong R / P \cong f_{j} R$, as rings, so $R / P$ and $f_{j} R$ have the same right uniform dimension. Inside $Q(S)$, the inclusion $T_{i j} \subset f_{i} S f_{j}=\sum_{v=1}^{n} f_{i} a_{v} f_{j} R$ leads to an inclusion $T_{i j}+J / J \hookrightarrow f_{i} S f_{j} / T_{i j} \cap J$ of $\left(f_{i} R, f_{j} R\right)$-bimodules. Denote $f_{i} a_{v} f_{j}$ by $x_{i}(1 \leqslant v$ $\leqslant n)$ and set $T_{i j} \cap J=Y$, this being an $\left(f_{i} R, f_{j} R\right)$-subbimodule of $f_{i} S f_{j}$. With denoting the coset in $f_{i} S f_{j} / Y$, choose $\Gamma=\Gamma(i, j)$, a subset of $\{1, \ldots, n\}$ maximal with respect to the property that $\bar{F}=\Sigma_{\gamma \in \Gamma} R f_{i} \bar{x}_{\gamma}$ is a free left $R f_{i}$-module with basis $\left\{\bar{x}_{\gamma} \mid \gamma \in \Gamma\right\}$. If $\left\{r_{\gamma} \mid \gamma \in \Gamma\right\} \subset R$ and $\Sigma_{\gamma \in \Gamma} r_{\gamma} x_{\gamma} \in T_{i j} \cap J$, then from the definition of $\Gamma$ it follows that $r_{\gamma} f_{i}=0$ for each $\gamma$. In particular, $F=\Sigma_{\gamma \in \Gamma} R x_{\gamma}=\sum_{\gamma \in \Gamma} R f_{i} x_{\gamma}$ is a free left $R f_{i}$-module of rank $|\Gamma|$, and $F \cap J=0$.

For $1 \leqslant v \leqslant n$, set $D_{v}=\left\{r \in R \mid r f_{i} x_{v} \in F+Y\right\}$, this being an ideal of $R$ since $f_{i} x_{v}$ normalizes $R$. It follows (from the definition of. $\Gamma$ ) that $P_{i} \subset D_{v}$, so $P_{i} \subset D=$ $\cap_{1}^{n} D_{v} \triangleleft R$ and $D f_{i} S f_{j} \subset F+Y$. Since $f_{i} S f_{j}$ is a torsion-free normalizing $\left(f_{i} R, f_{j} R\right)$ bimodule, there exists $[5,1.5] D^{\prime} \triangleleft R$ with $P_{j} \subset D^{\prime}$ and $f_{i} S f_{j} D^{\prime} \subset D f_{i} S f_{j}$. Then $\left(T_{i j}+J / J\right) D^{\prime} \subset F+Y+J / J \cong F / F \cap J=F$, this being an embedding of ( $\left.f_{i} R, f_{j} R\right)$-bimodules and $(R, R)$-bimodules. But $X_{i j}$ is right torsion-free over $f_{j} R$ (2.11), so $X_{i j} D^{\prime}$ is essential in $X_{i j}$ as a right $f_{j} R$-module. Therefore u. $\operatorname{dim} .\left(X_{i j}\right)_{R}=$ u. $\operatorname{dim} .\left(X_{i j} D^{\prime}\right)_{R} \leqslant$ u. $\operatorname{dim} .\left(F_{R}\right)=|\Gamma|$ u. $\operatorname{dim} .[R / P]_{R}$, the last equality following because $F_{R}$ is a direct sum of $|\Gamma|$ copies of $f_{j} R$.

The next result encompasses both a result of Lanski [10] and one of Stewart [16].

THEOREM 6.3. If $P, J$ is a linked pair of prime ideals then $R / P$ is right Goldie if and only if $T / J$ is right Goldie.

Proof. Since prime right Goldie rings are characterized as prime right nonsingular rings with finite right uniform dimension, this follows at once from 6.1 and 6.2.

In the case where $R / P$ and $T / J$ are right Goldie, Theorem 6.2 can be strengthened by showing that u.dim. $(T / J)_{T}$ is an integer multiple of u.dim. $(R / P)_{R}$, thus providing another instance of the validity of the "additivity principle". First, some additional notation is required.

Notation 6.4. With $R \subset T \subset S$ providing a standard setting for $J$, and $P, J$ a linked pair, suppose $\operatorname{conn}_{R}(J)=\left\{P_{1}, \ldots, P_{k}\right\}$. Denote by $R^{\#}$ the subring $\Sigma_{i=1}^{\oplus k} f_{i} R$ of $Q(S)$. Via the homomorphism induced by $f_{i} \mapsto \bar{f}_{i}, R^{\#}$ embeds as a subring of $Q(T / J)$ with the same unity, and so $T^{\circ}+J / J$ can be regarded as an $R^{\#}$-bimodule. Since $T^{\circ}+J / J=\sum_{i, j=1}^{\oplus k}\left(T_{i j}+J\right) / J$ and each $T_{i j}+J / J$ is torsion-free as an $\left(f_{i} R, f_{j} R\right.$ )-bimodule, from $3.6, T^{\circ}+J / J$ is torsion-free as an $R^{\#}$-bimodule. 
Next, a preparatory lemma is needed.

LEMMA 6.5. In the embedding of $R^{\#}$ into $Q(T / J)$, a regular element $c$ of $R^{\#}$ is not a zero-divisor in $Q(T / J)$.

Proof. Since $Q(T / J)$ is the Martindale quotient ring of $T^{\circ}+J / J$, it is sufficient to show that rt.ann. ${ }_{\left(T^{\circ}+J / J\right)}(c)=$ It. ann. ${ }_{\left(T^{\circ}+J / J\right)}(c)=0$. Furthermore, we may write $c=\sum_{1}^{k} c_{i}$ with $c_{i}$ a regular element of $f_{i} R$.

Suppose first that $t \in T^{\circ}$ and $c(t+J)=0$. By 3.1,

$$
\left(T^{\circ}+J\right) / J=\sum_{i, j=1}^{k} \oplus\left(T_{i j}+J\right) / J,
$$

so we may write $t+J=\Sigma_{i, j}\left(t_{i j}+J\right)$ with $t_{i j} \in T_{i j}$. Then $c(t+J)=0$ implies $c_{i} t_{i j} \in J$ for each $i$ and $j$.

Suppose $i$ and $j$ are chosen, and let $\Gamma=\Gamma(i, j), \bar{F}$, and $0 \neq D^{\prime} \triangleleft f_{j} R$ be chosen as in the proof of 6.2(ii). Then $\bar{F}$ is an $\left(f_{i} R, f_{j} R\right)$-bimodule, free as an $f_{i} R$-module, with an $\left(f_{i} R, f_{j} R\right)$-normalizing basis $\left\{\bar{x}_{\gamma} \mid \gamma \in \Gamma\right\}$. Furthermore, as in $6.2,\left(T_{i j}+J / J\right) D^{\prime}$ $\subset \bar{F}$. For any $d$ in $D^{\prime}$, write $\left(t_{i j}+J\right) d=\Sigma_{\gamma} r_{\gamma} \bar{x}_{\gamma}$ with $r_{\gamma} \in f_{i} R$. Then $c_{i}\left(t_{i j}+J\right) d=$ $0=\sum_{\gamma} c_{i} r_{\gamma} \bar{x}_{\gamma}$, and since $f_{i} \mathrm{R} F$ is free, $c_{i} r_{\gamma}=0$ for each $\gamma$. Since $c_{i}$ is regular in $f_{i} R$, each $r_{\gamma}=0$ : therefore $\left(t_{i j}+J\right) D^{\prime}=0$, and since $\left(T_{i j}+J\right) / J$ is right torsion-free over $f_{j} R, t_{i j}+J=0$.

Thus $t+J=0$ whenever $c(t+J)=0$, and so $c$ is right regular in $Q(T / J)$. A symmetrical argument (including a left-hand version of 6.2 ) completes the proof.

Now comes the "additivity principle".

THEOREM 6.6. Assume that $P, J$ is a linked pair of prime with $T / J$ and (equivalently) $R / P$ right Goldie. Then

(i) the embedding of $R^{\#}$ into $Q(T / J)$ gives an embedding of $Q_{c}\left(R^{\#}\right)$ into $Q_{c}(T / J)$, where $Q_{c}$ is the classical right quotient ring, and

(ii) u. $\operatorname{dim} .(T / J)_{T}=z \cdot$ u. $\operatorname{dim} .(R / P)_{R}$ where $z$ is an integer divisible by $k=$ $\left|\operatorname{conn}_{R}(J)\right|$.

Proof. (i) As usual, a standard setting for $J$ may be assumed, and $I_{0}$ is the ideal of $S$ described previously. Then each of $I_{0} \cap T+J / J, R^{\#}+T^{\circ} / J$, and $T / J$ are orders in $Q_{c}(T / J)$. By 6.5, any regular element of $R^{\#}$ remains regular as an element of $R^{\#}+T^{\circ} / J$, and hence is a unit in $Q_{c}(T / J)$, so $Q_{c}\left(R^{\#}\right)$ embeds in $Q_{c}(T / J)$.

(ii) It now follows by standard arguments (see [18, Lemma 1 or 9,3.8]) that u. $\operatorname{dim} .[T / J]_{T}$ is a multiple of the composition length of $Q_{c}\left(R^{\#}\right)$, and the latter equals $k\left(\operatorname{rank}(R / P)_{R}\right)$.

REMARKS. (1) This resolves a question left open in [9].

(2) If $P, J$ and $I$ are primes of $R, T$ and $S$, respectively, with $J, I$ forming a standard pair and $P$ linked to both $J$ and $I$, then (when $R / P, T / J$ and $S / I$ are right Goldie) $\operatorname{rank}(R / P)_{R} \operatorname{divides} b o t h \operatorname{rank}(T / J)_{T}$ and $\operatorname{rank}(S / I)_{S}$. It need not be true that $\operatorname{rank}(T / J) \operatorname{divides} \operatorname{rank}(S / I)$. For example, let $A$ be any commutative domain 
with a surjective homomorphism $\theta: A \rightarrow A$ with nonzero kernel $K$. Take $S=M_{3}(A)$, $R$ the subring $\{\operatorname{diag}(a, a, \theta(a)) \mid a \in A\}$ and $T$ the subring consisting of all elements of the form

$$
\left[\begin{array}{ccc}
a & b & 0 \\
c & d & 0 \\
0 & 0 & \theta(d)
\end{array}\right] \quad \text { with } a, b, d \in A \text { and } c \in K
$$

Then taking $J=0$ (in $T$ ) and $I=0$ (in $S$ ), it is easily verified that $\operatorname{rank}\left(T_{T}\right)=2$ and $\operatorname{rank}\left(S_{S}\right)=3$.

Before considering the passage of other chain conditions, and Krull dimension, between $T / J$ and $R / P$, a preparatory result is required.

Proposition 6.7. Suppose that $T$ /ann $M$ is right or left Noetherian, and that $M_{T}$ is a Noetherian module with $\kappa(M)=\alpha$. Then $M_{R}$ is Noetherian with $\kappa\left(M_{R}\right)=\alpha$.

Proof. Suppose by Noeiherian induction that $M$ is a counterexample of minimal possible Krull dimension and that no proper factor module of $M_{T}$ is also a counterexample. Any critical submodule of $M_{T}$ will also have this property, so suppose $M$ is indeed critical. Since $T /$ ann $M$ is Noetherian, $M$ has a prime cyclic submodule $M^{\prime}$, with prime annihilator, $J$ say. Again, we may suppose $M=M^{\prime}$; and without loss, we may also suppose the setting to be standard for $J$.

Suppose, then, that $M=T / K$. By 2.9, $0 \neq\left(I_{0} \cap T\right)+K / K \subset \Sigma^{\oplus} X_{i} \subset T / K=$ $M$ with each $X_{i}$ either zero or a prime uniform $T_{i}$-module. Suppose $0 \neq Y \triangleleft\left(X_{i}\right)_{T}$; we will show that $\left(X_{i} / Y\right)_{R}$ is Noetherian, with $\kappa\left(X_{i} / Y\right)_{R}<\alpha$.

To see this, say $Y \simeq Z / K$, with $K \subset Z \subset T T^{\circ} f_{i}+K$. There is then a well-defined multiplication $Y \cdot T^{\circ} \subset T / K$ under which

$$
Y\left(I_{0} \cap T\right) \subset Y \oplus \sum_{\substack{j=1 \\ j \neq i}}^{m} X_{j}
$$

(since $\left.Y T_{i i} \subset Y\right)$. Now

$$
X_{i} / Y \simeq\left(X_{i} \oplus \sum_{j \neq i} X_{j}\right) /\left(Y \oplus \sum_{j \neq i} X_{j}\right) \subseteq M /\left(Y \oplus \sum_{j \neq i} X_{j}\right),
$$

which is a homomorphic image of $\left(M / Y\left(I_{0} \cap T\right)\right)_{R}$. By induction, the latter is Noetherian, with Krull dimension $<\alpha$, so the same is true of $\left(X_{i} / Y\right)_{R}$.

Considering $X_{i}$ as an $R$-module, or equivalently as an $f_{i} R$-module, B.13 of Appendix B shows that u. $\operatorname{dim} .\left(X_{i}\right)_{R}<\infty$ and that any essential submodule $E_{R}$ contains a nonzero $T_{i}$-submodule, so from the preceding paragraph it follows that $\left(X_{i} / E\right)_{R}$ is Noetherian and has Krull dimension $<\alpha$. Therefore, by [4, Lemma 1], $\left(X_{i}\right)_{R}$ is Noetherian and $\kappa\left(X_{i}\right)_{R} \leqslant \alpha$. Now both $M / M\left(I_{0} \cap T\right)_{R}$ and $M\left(I_{0} \cap T\right)_{R}$ are Noetherian, with Krull dimension $\leqslant \alpha$, so the same is true of $M_{R}$. This completes the proof.

THEOREM 6.8. If $P, J$ is a linked pair of primes then $R / P$ is right Noetherian with Krull dimension $\alpha$ if and only if the same is true of $T / J$. 
Proof. Without loss, suppose the setting is standard for $J$. Now if $R / P$ is right Noetherian then so are $R$ (as noted earlier), $S_{R}\left(=\sum_{1}^{n} a_{i} R\right.$ ), and $(T / J)_{R}$. Thus $T / J$ is right Noetherian. Whereas if $T / J$ is right Noetherian then so is $(T / J)_{R}$ (by 6.7), and hence $R / J \cap R$ and $R / P$ are right Noetherian. The Krull dimension is dealt with similarly.

Another immediate consequence of 6.7 is the following, which is also, however, an easy consequence of the corresponding result for $R, S$.

Corollary 6.9. $R$ is right Noetherian or right Artinian if and only if the same is true of $T$.

Appendix B. This section concerns a chain $R \subset T \subset S \subset M \subset Q=Q(S)$ where $R$ and $S$ are both prime rings, $T$ an intermediate ring, $M$ a normalizing $R$-bimodule with generators $x_{1}, \ldots, x_{n}$, and $Q$, the Martindale ring of right quotients of $S$, is a (right and left) torsion-free $R$-bimodule. Moreover, $J$ is a prime ideal of $T$ with $J \cap R=0$.

The results obtained here are all used in earlier sections for the chain described in §2, viz. $f_{i} R \subset T_{i} \subset S_{i} \subset f_{i} S f_{i} \subset Q\left(S_{i}\right)=f_{i} Q(S) f_{i}$; and the statements of such "applications" will be given in that earlier notation. That apart, the notation will remain as in the first paragraph above.

This appendix is a continuation of Appendix A of [7], the approach taken here being based on ideas from $[9,15$, and 16]. Now in Appendix $A$ it was shown that the embedding $R \subset S$ extends to an embedding of $N R$ (the normal closure of $R$ ) into $Q$. With $\Re$ denoting $\{q \in Q \mid q R=R q\}$, and $Q$ the subring of $Q$ generated by $\Re$ and $R$, each $H \triangleleft_{R} Q_{R}$ gives rise to a certain subset $\bar{H}$ of $Q$ (defined in [7]). The relevant properties are as follows.

Proposition B.1. With $H, K$ and $L$ denoting subbimodules of ${ }_{R} S_{R}$, and $\mathfrak{\Re}_{H}$ denoting $\bar{H} \cap \Re$,

(i) if $q \in \bar{H}$ then $q Y+Y^{\prime} q \subset H$ for some nonzero ideals $Y$ and $Y^{\prime}$ of $R$, and if $q \in \mathbb{Q}$ satisfies $Y q Y^{\prime} \subset H$ for $0 \neq Y, Y^{\prime} \triangleleft R$, then $q \in \bar{H}$;

(ii) $\bar{H}=\Re_{H} R$, this being an $R$-bimodule and an NR-bimodule;

(iii) $\bar{H}$ is a free right and left NR-module of finite rank, and a finite NR-normalizing basis may be found in $\Re_{H}$. Any maximal $R$-independent subset of $\Re_{H}$ comprises such a basis;

(iv) if $H K \subset L$, then $\bar{H} \bar{K} \subset \bar{L}$. In particular, $\bar{T}=\mathfrak{N}_{T} R$ is a subring of $\bar{S}=\Re_{S} R$ containing $\mathfrak{R}_{R} R=N R$, and $\bar{B} \triangleleft \bar{T}$ whenever $B \triangleleft T$.

Proposition B.2. $\Re_{J} R$ is maximal among ideals $I$ of $\Re_{T} R$ satisfying $I \cap T=J$.

Proof. $J \subset M \subset Q$ so $J \subset \bar{J} \cap T=\mathfrak{R}_{J} R \cap T$, and if $x \in \mathfrak{M}_{J} R \cap T$ then $0 \neq$ $Y x \subset J$ (by torsion-freeness of $Q$, and B.1), so $J$ is essential in ${ }_{R}\left(T \cap \mathfrak{N}_{J} R\right)_{R}$. By [6, 2.2], there exists $0 \neq D \triangleleft R$ with $D\left(T \cap \Re_{J} R\right) \subset J$. Since $D \not \subset J$ (because $J \cap R$ $=0$ ), it follows that $T \cap \Re_{T} R \subset J$, so $J=T \cap \Re_{J} R$.

Suppose now that $\mathfrak{N}_{J} R \subset L \triangleleft \mathfrak{N}_{T} R$ with $L \cap T \subset J$. Then for $x$ in $L\left(\subset \mathfrak{N}_{T} R\right)$ there exists $0 \neq Y \triangleleft R$ with $Y x \subset T \cap L \subset J$; hence $x \in \bar{J}=\Re_{T} R$ and thus $\Re_{J} R=L$. 
Corollary B.3. $\mathcal{M}_{J} R$ is a prime ideal of $\mathfrak{M}_{T} R$ and $\mathfrak{M}_{J} R \cap N R=0$.

Proof. That $\mathfrak{M}_{J} R$ is prime follows from B.2. If $x \in \Re_{J} R \cap N R$, then there exists $0 \neq Y \triangleleft R$ with $Y x \subset J \cap R=0$ and since ${ }_{R} Q_{R}$ is torsion-free, $x=0$.

Proposition B.4. If $J \underset{\neq}{\subset} B \triangleleft T$, then $B \cap R \neq 0$.

Proof. Since $J \subset B \subset T \cap \mathfrak{M}_{B} R, \mathfrak{N}_{J} R$ is a proper subset of $\mathfrak{M}_{B} R \triangleleft \mathfrak{M} \mathbb{R}_{T} R$. Since $\mathfrak{N}_{T} R$ is a normalizing extension of $N R$, the "incomparability" theorem [5, 5.10] implies $\mathfrak{M}_{B} R \cap N R \neq 0$. If $0 \neq x \in \mathfrak{K}_{B} R \cap N R$, then $0 \neq D x \subset B \cap R$ for some $D \triangleleft R$.

Application B.5. With notation as in 3.7, $J_{i}$ is maximal among ideals of $T_{i}$ meeting $f_{i} R$ at zero.

Lemma B.6. Let $b_{1}, \ldots, b_{v}$ be any $N R$-basis for $\mathfrak{T}_{T} R$ whose elements normalize $R$. Then there exist nonzero ideals $D$ and $F$ of $R$ for which $\Lambda=\sum_{1}^{v} F b_{i}$ is a subring (without 1) of $T$ containing $T D T$.

Proof. Since $\mathfrak{O R}_{T} R$ is a ring, each product $b_{i} b_{j}$ may be written $b_{i} b_{j}=\sum_{k=1}^{v} \rho_{i j k} b_{k}$ with $\rho_{i j k} \in N R$. Then $F_{1} \rho_{i j k} \subset R$ (for all $i, j, k$ ) for some $0 \neq F_{1} \triangleleft R$. Choose $F_{1 j} \triangleleft R$ with $b_{j} F_{1 j}=F_{1} b_{j}(\neq 0)$, for each $j$, and $Z \triangleleft R$ with $0 \neq Z b_{j} \subset T$ (using B.1(i)); and set $F=Z \cap\left(\cap_{j=1}^{v} F_{1 j}\right)$. Then it follows that $F b_{i} F b_{j} \subset \sum_{k=1}^{v} F b_{k} \subset T$, so $\Lambda=\Sigma_{1}^{v} F b_{i}$ is a ring. Since ${ }_{R} N R_{R}$ is uniform, the $(R, R)$-bimodule ranks of $T$, $\mathbb{Q}_{T} R$ and $\Lambda$ all equal $v$, so $\Lambda$ is essential in ${ }_{R} T_{R}$. Using the torsion-freeness of ${ }_{R} Q_{R}$ and applying [6, 2.2] (twice) there exist $D, D^{\prime} \triangleleft R$ with $0 \neq D T \subset T D^{\prime} \subset \Lambda$, and so $T D T \subset \Lambda$.

REMARK. Suppose that $H_{1} \subset H_{2} \subset \cdots \subset H_{n}$ was a finite chain of subrings (with or without 1) of $S$ with $R H_{i} R \subset H_{i}$. From B.1, a chain $B_{1} \subset B_{2} \subset \cdots \subset B_{n}$ of finite subsets of $\Re_{S}$ could be chosen with $B_{i}$ an $R$-normalizing set and an $N R$-basis for $\bar{H}_{i}$. Minor modifications of the above proof would yield ideals $E$ and $D$ of $R$ with $\Lambda_{i}=\Sigma_{b \in B_{i}} F b$ a ring of $H_{i}$, and $H_{i} D H_{i} \subset \Lambda_{i}$.

The next few results are concerned with a right $T$-module $X$ which, when viewed as a right $R$-module, is torsion-free. The notation $\left\{b_{1}, \ldots, b_{v}\right\}, F$ and $D$ of B.6 will be retained.

Lemma B.7. Let $Y \triangleleft X_{R}$ and set $Y_{i}=\left\{x \in X F \mid x b_{i} \subset Y\right\}$ and $\beta(Y)=\bigcap_{1}^{v} Y_{i}$. Then

(i) $Y_{i}$ and $\beta(Y) \triangleleft X F_{R}$,

(ii) the map $x \mapsto x b_{i}$ from $X F$ to $X$ induces an embedding $\mathcal{L}\left(X F / Y_{i}\right)>\mathscr{L}(X / Y)$ of lattices of $R$-submodules and

(iii) if $Y$ is essential in $X_{R}$ then $Y_{i}$ and $\beta(Y)$ are essential in $X F_{R}$.

Proof. (i) is clear and (ii) is easily verified, since $b_{i} R=R b_{i}$.

(iii) Suppose $0 \neq A \triangleleft X F_{R}$. If $A b_{i}=0$ then $A \subset Y_{i}$, while if $A b_{i} \neq 0$ then $A b_{i} \cap Y$ $\neq 0$ and then $A \cap Y_{i} \neq 0$. Therefore $Y_{i}$ is essential for each $i$, and then so is $\beta(Y)$.

Proposition B.8 (CF. [16, 24]). If $Y_{R}$ is essential in $X_{R}$ then $Y \supset H \triangleleft X_{T}$ with $H_{R}$ essential in $X_{R}$. 
Proof. Since $X_{R}$ is torsion-free, $X^{\prime} E$ is essential in $X_{R}^{\prime}$ whenever $0 \neq E \triangleleft R$ and $X^{\prime} \triangleleft X_{R}$. Now consider the chain

$$
\beta(Y) D \subset \beta(Y) T D T \subset \beta(Y) \Lambda=\beta(Y)\left(\sum_{1}^{v} F b_{i}\right) \subset Y .
$$

Since $\beta(Y) D_{R} \subset \beta(Y)_{R} \subset Y_{R}$, with each essential in the next, choose

$$
H=\beta(Y)(T D T) \text {. }
$$

Lemma B.9. (i) $X_{R}$ contains a submodule $Y_{R}$ maximal with respect to $\beta(Y)=0$.

(ii) For any $Y \triangleleft X_{R}, \beta(Y) \neq 0$ if and only if $Y$ contains a nonzero T-submodule of $X$.

Proof. (i) This follows by a routine application of Zorn's lemma.

(ii) If $\beta(Y) \neq 0$ then $0 \neq \beta(Y) T D T \subset Y$, as in the previous proof. Conversely, if $0 \neq H_{T} \subset Y$ then $H F b_{i} \subset H T \subset Y$ for all $i$, so $0 \neq H F \subset \beta(Y)$.

Proposition B.10. Let $Y \triangleleft X_{R}$ be maximal with respect to $\beta(Y)=0$, and suppose u. $\operatorname{dim} .\left(X_{T}\right)=m<\infty$. Then

(i) u. $\operatorname{dim} .(X / Y)_{R} \leqslant m$ and

(ii) u. $\operatorname{dim} .\left(X_{R}\right) \leqslant m v\left(\right.$ where $v=$ u. $\left.\operatorname{dim} .{ }_{R} T_{R}\right)$.

Proof. (i) Suppose $Y \subset A_{i} \triangleleft X_{R}$ with $\Sigma_{1}^{t}\left(A_{i} / Y\right)$ a direct sum in $X / Y$. For each $i$, $\beta\left(A_{i}\right) \neq 0$ and $A_{i}$ contains $0 \neq\left(B_{i}\right)_{T}$, by B.9. If $t>m$, then

$$
B=B_{j} \cap\left(\sum_{\substack{i=1 \\ i \neq j}}^{t} B_{i}\right) \neq 0
$$

for some $j$, and $B \subset A_{j} \cap\left(\sum_{i \neq j} A_{i}\right)=Y$, so $\beta(B)=0$, contradicting B.9. Therefore $t \leqslant m$, proving (i).

(ii) Since $0=\beta(Y)=\bigcap_{1}^{v} Y_{i}, X F_{R}$ embeds in $\amalg_{1}^{v}\left(X F / Y_{i}\right)$. But, by B.7(ii), u. $\operatorname{dim} .\left(X F / Y_{i}\right)_{R} \leqslant$ u. $\operatorname{dim} .(X / Y)_{R}$ and so u. $\operatorname{dim} .\left(X F_{R}\right) \leqslant m v$. But $X F$ is essential in $X_{R}$ (by the torsion-freeness of $X_{R}$ ), and so u. $\operatorname{dim} .\left(X_{R}\right) \leqslant m v$.

CorollaRY B.11. (i) If $X_{T}$ is uniform then u. $\operatorname{dim} .\left(X_{R}\right) \leqslant \mathrm{u} . \operatorname{dim} .\left({ }_{R} T_{R}\right) \leqslant n$;

(ii) If $X_{T}$ is simple then $X_{R}$ is semisimple with composition length at most $n$;

(iii) if $(T / J)_{T}$ has finite uniform dimension, so do $(T / J)_{R}$ and $R_{R}$; and

(iv) if $T / J$ is prime right Goldie then $R$ is prime right Goldie.

Proof. (i) This is simply B.10(ii) for $m=1$.

(ii) Suppose $X_{T}$ is simple. With notation as in B.10, if $0 \neq A / Y \triangleleft X / Y$ then $\beta(A) \neq 0$ and then (using B.9) $A=X$ : therefore $(X / Y)_{R}$ is simple and so by B.7, each $\left(X F / Y_{i}\right)_{R}$ is simple or zero. Therefore $X F$ is semisimple of finite length. But $X F$ is essential in $X_{R}$, so it follows (from B.8) in this case that $X F=X$.

(iii) This follows immediately from B.10(ii), with $X_{T}=(T / J)_{T}$. To verify that $(T / J)_{R}$ is torsion-free, note that if $t \in T$ and $0 \neq D \triangleleft R$ with $t D \subset J$, then (since ${ }_{R} T_{R}$ is torsion-free) $T D^{\prime} \subset D T$ for some nonzero ideal $D^{\prime}$ of $R$, by [6, 2.2]. Then $t T D^{\prime} \subset J$, with $D^{\prime} \not \subset J$, and so $t \in J$. 
(iv) This follows from (iii) and the fact that $R$ inherits a.c.c. on right annihilators from $T / J$.

APPliCation B.12. With notation as in 4.6, each $X_{i}$ is semisimple with composition length at most $n$.

ApPlication B.13. With notation as in 6.2 and 6.7,

(i) the uniform dimension of $X_{i}$, as an $f_{i} R$-module, is at most $n$;

(ii) any essential $R_{i}$ submodule of $X_{i}$ contains a nonzero $T_{i}$-submodule;

(iii) if $T_{i} / J_{i}$ has finite right uniform dimension, then so does $f_{i} R$;

(iv) if $T_{i} / J_{i}$ is prime right Goldie, then so too is $f_{i} R$.

The proof of the next result is an adaptation of that of $[16,3.2]$.

THEOREM B.14. With $Z$ the inverse image in $T$ of the right singular ideal of $T / J$, and $Z(R)$ the (right) singular ideal of $R$, then $Z \cap R=Z(R)$.

Proof. ${ }_{R}(M / J)_{R}$ is a normalizing $(R, R)$-bimodule generated by the cosets $\bar{x}_{i}=x_{i}+J$. Let $\Gamma$ be a subset of $\{1, \ldots, n\}$ maximal with respect to the property that $\Sigma_{\gamma \in \Gamma} R \bar{x}_{\gamma}$ is a free left $R$-module with $\left\{\bar{x}_{\gamma} \mid \gamma \in \Gamma\right\}$ as basis. Then, as in [16, 2.1], it follows that

(i) ${ }_{R} F=\Sigma_{\gamma \in \Gamma} R x_{\gamma}$ is free,

(ii) if $\left\{r_{\gamma} \mid \gamma \subset \Gamma\right\} \subset R$ with $\Sigma r_{\gamma} x_{\gamma} \in J$ then each $r_{\gamma}=0$,

(iii) $D M \subset F+J$ for some $0 \neq D \triangleleft R$, and

(iv) $F \cap J=0$.

Since ${ }_{R} M_{R}$ is torsion-free, there exists $0 \neq D^{\prime} \triangleleft R$ with $T D^{\prime} \subset D T$.

Suppose first that $r \in Z(R)$, and suppose $0 \neq(K / J) \triangleleft_{\mathrm{rt}} T / J$. Then $K D^{\prime} T \subset$ $T D^{\prime} T \subset D T \subset F+J$, but $K D^{\prime} T \not \subset J$ (since $J \cap R=0$ and $K \not \subset J$ ) so every element of $K D^{\prime} T$ may be written $y=\sum_{\gamma \in \Gamma} r_{\gamma} x_{\gamma}+w$ with $r_{\gamma} \in R, w \in J$. Choosing an element $y$ not in $J$ for which, in such a representation, $\left\{\gamma \mid r r_{\gamma}=0\right\}$ is as large as possible, it follows (for details, see [16]), that $y \in K D^{\prime} T \sim J$ but $r r_{\gamma}=0$ for each $\gamma$, so $r y \in J$. It now follows that $r \in Z$.

Conversely, suppose $r \in R \cap Z$ and let $0 \neq K \triangleleft_{\mathrm{rt}} R$. Then $K D T \subset K F+J$ but $K D T \not \subset J$ (otherwise $K D \subset J \cap R=0$ ). Since $r \in Z$ there exists an element $y$ in $K D T \sim J$ with $r y \in J$. Writing $y=\Sigma_{\gamma} k_{\gamma} x_{\gamma}+w\left(k_{\gamma} \in K, w \in J\right), r(y-w) \in$ $F \cap J=0$ so $r k_{\gamma}=0$ for each $\gamma$. Since $y \notin J, k_{\gamma} \neq 0$ for some $\gamma$ (by the definition of $\Gamma$ ) and so $K \cap \mathrm{rt}$. ann. ${ }_{R}(r) \neq 0$. This shows $r \in Z(R)$.

APPLICATION B.15. With the notation of $6.1, Z_{i} \cap f_{i} R=Z\left(f_{i} R\right)$, the right singular ideal of $f_{i} R$.

\section{REFERENCES}

1. S. A. Amitsur, Rings of quotients and Morita contexts, J. Algebra 17 (1971), 273-298.

2. J. Bit-David and J. C. Robson, Normalizing extensions. I, Ring Theory 1980 (F. Van Ostaeyen, ed.), Lecture Notes in Math., vol. 825, Springer-Verlag, Berlin and New York, 1980.

3. J. Bit-David, Normalizing extensions. II, Ring Theory 1980 (F. Van Ostaeyen, ed.), Lecture Notes in Math., vol. 825, Springer-Verlag, Berlin and New York, 1980.

4. E. Formanek and A. V. Jategaonkar, Subrings of Noetherian rings, Proc. Amer. Math. Soc. 46 (1974), 181-186.

5. A. G. Heinicke and J. C. Robson, Normalizing extensions: prime ideals and incomparability, J. Algebra 72 (1981), 237-268. 
6. __ Normalizing extensions: nilpotency, J. Algebra 76 (1982), 459-470.

7. N__ Normalizing extensions: prime ideals and incomparability. II, J. Algebra (to appear).

8. S. Jabbour, Intermediate normalizing extensions, Comm. Algebra (to appear).

9. Chain conditions in intermediate normalizing extensions, Comm. Algebra (to appear).

10. C. Lanski, Goldie conditions in finite normalizing extensions, Proc. Amer. Math. Soc. 79 (1980), 515-519.

11. M. Lorenz, Finite normalizing extensions of rings, Math. Z. 176 (1981), 447-484.

12. W. K. Nicholson and J. F. Watters, Normal classes of rings and tensor products, Comm. Algebra 9 (1981), 299-311.

13. D. S. Passman, Prime ideals in normalizing extensions, J. Algebra 73 (1981), 556-572.

14. R. Resco, Radicals of finite normalizing extensions, Comm. Algebra 7 (1981), 713-725.

15. J. C. Robson, Prime ideals in intermediate extensions, Proc. London Math. Soc. (3) 44 (1982), 372-384.

16. P. N. Stewart, Properties of intermediate extensions, Comm. Algebra (to appear).

17. P. N. Stewart and J. F. Watters, Properties of normalizing extensions and fixed rings, Comm. Algebra (to appear).

18. R. B. Warfield, Jr., Prime ideals in ring extensions, J. London Math. Soc (2) (to appear).

Department of Mathematics, University of Western Ontario, London, Ontario, Canada N6A 5B7

Department of Mathematics, University of LeEDS, LeEDS, ENGLAND 